Published in final edited form as:

Sci Signal. ; 11(519): . doi:10.1126/scisignal.aao4220.

\title{
Convergence of Wnt, growth factor and trimeric G protein signals on Daple
}

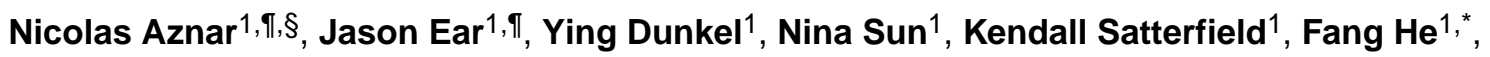 \\ Nicholas Kalogriopoulos ${ }^{1}$, Inmaculada Lopez-Sanchez ${ }^{1}$, Majid Ghassemian ${ }^{2}$, Debashis \\ Sahoo $^{3,4}$, Irina Kufareva ${ }^{5}$, and Pradipta Ghosh ${ }^{1,6,7, \S}$ \\ ${ }^{1}$ Department of Medicine, University of California, San Diego, La Jolla, California 92093, USA \\ ${ }^{2}$ Department of Chemistry and Biochemistry, University of California, San Diego, La Jolla, \\ California 92093, USA \\ ${ }^{3}$ Skaggs School of Pharmacy and Pharmaceutical Sciences, University of California, San Diego, \\ La Jolla, California 92093, USA \\ ${ }^{4}$ Department of Computer Science and Engineering, Jacob's School of Engineering, University of \\ California, San Diego, La Jolla, California 92093, USA \\ ${ }^{5}$ Department of Pediatrics, University of California, San Diego, La Jolla, California 92093, USA \\ ${ }^{6}$ Department of Cellular and Molecular Medicine, University of California, San Diego, La Jolla, \\ California 92093, USA \\ ${ }^{7}$ Moores Cancer Centre, University of California, San Diego, La Jolla, California 92093, USA
}

\section{Abstract}

Cellular proliferation, differentiation, and morphogenesis are shaped by multiple signaling cascades; their concurrent dysregulation plays an integral role in cancer progression and is a common feature of many malignancies. Three such cascades that contribute to the oncogenic potential are those mediated by Wnt and Frizzled (FZD), growth factor receptor tyrosine kinases (RTKs), and trimeric G proteins and GPCRs. Daple is a Dishevelled (Dvl)- and FZD-binding protein and a guanine-nucleotide exchange factor $(\mathrm{GEF})$ for the trimeric $\mathrm{G}$ protein, $\mathrm{G}_{\mathrm{ai}}$. Daple enhances $\beta$-catenin-independent Wnt signaling through its ability to activate the pathway downstream of the Wnt5/FZD7 pathway. Here we identified that Daple is a substrate of multiple RTKs and non-RTKs, and hence, a point of convergence for all three cascades. We show that

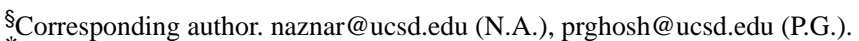

* Current address: Center of Infectious Diseases, West China Hospital and Division of Infectious Diseases, State Key Laboratory of Biotherapy, Sichuan University, Chengdu, Sichuan, PR China.

IThese authors equally contributed to this work.

AUTHOR CONTRIBUTIONS

N.A, J.E and P.G designed, performed and analyzed most of the experiments in this work. Y.D. cloned and generated all Daple mutants used in this work. N.S, F.H and K.S assisted with protein expression, purification, and in carrying out protein-protein interaction assays; I.L-S carried out in vitro kinase assays; N.K performed in vitro kinase assays; M.G performed the mass spectrometry analyses. D.S and P.G analyzed the patient datasets using the Hegemon software; I.K generated the homology model for Daple-bound Dvl and provided structure-based guidance to study Daple mutants. N.A and P.G conceived the project and wrote the manuscript. P.G supervised and funded the project.
}

COMPETING FINANCIAL INTERESTS

The authors declare no competing financial interests. 
phosphorylation by both RTKs and non-RTKs near the Dvl-binding motif in Daple dissociated Daple:Dvl complexes and augmented the ability of Daple to bind and activate $\mathrm{G}_{\mathrm{ai}}$ which potentiated $\beta$-catenin-independent Wnt signals and triggered epithelial-mesenchymal transition (EMT) in a manner similar to that triggered by Wnt5A/FZD. Although Daple acts as a tumor suppressor in the healthy colon, but concurrent upregulation of Daple and epidermal growth factor receptor (EGFR) in colorectal tumors from patients was associated with poor prognosis. We conclude that Daple-dependent activation of $\mathrm{G}_{a \mathrm{i}}$ and enhancement of $\beta$-catenin-independent Wnt signals is not just triggered by Wnt5a/FZD to suppress tumorigenesis, but also is hijacked by growth factor-RTKs to stoke tumor progression. Thus, this work defines a crosstalk paradigm amongst growth factor RTKs, trimeric G-proteins, and Wnt/FZD in cancer biology.

\section{Introduction}

Molecular characterization of tumors has revealed that multiple signaling pathways are often simultaneously dysregulated in cancer cells. Although each of these pathways are often conceptualized as independent entities, their complex crosstalk shapes many aspects of cancers, including proliferation, invasion, immune evasion, chemoresistance and stemness (1-7). Aberrant Wnt/Frizzled, heterotrimeric (trimeric) $G$ proteins/G protein-coupled receptors [GPCRs] and growth factor/receptor tyrosine kinase [RTK]-based signaling cascades are three examples of such pathways that are frequently dysregulated in cancers, and the crosstalk between these pathways are of paramount importance in driving several properties of cancer cells. For example, aberrant activation of $\beta$-catenin-dependent Wnt signals [the so-called canonical $\beta$-catenin-TCF/LEF transcriptional program] secondary to adenomatous polyposis coli (APC), axin, and $\beta$-catenin gain-of-function mutations are associated with the development of colon cancer, desmoid tumors, gastric cancer, hepatocellular carcinoma, medulloblastoma, melanoma, ovarian cancer, pancreatic cancer, and prostate cancer [reviewed in (1)]. However, these mutations alone do not account for the observed $\beta$-catenin hyperactivity; instead, it is the crosstalk between the growth factor RTK and the $\beta$-catenin-dependent $\mathrm{Wnt} /$ Frizzled pathways that synergistically hyperactivate the $\beta$ catenin-dependent transcriptional program $(2,8)$. This crosstalk between growth factor/RTK and $\mathrm{Wnt} / \beta$-catenin pathways is a well-defined paradigm that is frequently encountered in cancers and enables growth factor RTKs to potentiate $\beta$-catenin signaling through distinct mechanisms [reviewed in $(2,8)$ ]: (i) by triggering PI3K-Akt signals, which in turn can inhibit the downstream kinase, GSK3 $\beta$; inhibition of GSK3 $\beta$ prevents proteasomal degradation of $\beta$-catenin and results in increased accumulation of $\beta$-catenin, followed by nuclear localization; activated Akt can also directly phosphorylate $\beta$-catenin and enhance its transcriptional activity (9); (bii) by triggering the MAPK/ERK kinase cascade, which can stabilize $\beta$-catenin by evading proteasomal degradation (10-13); and finally, (iii) by increased shedding of $\beta$-catenin from E-cadherin-bound junctional complexes $(14,15)$. These mechanisms underscore the importance of concurrent aberrant signaling [triggered by sequential genetic/epigenetic 'hits']; in solid tumors, aberrations in as few as three driver genes or pathways appear to suffice for a cell to evolve into an advanced cancer (16).

Although the elaborate crosstalk between growth factors and the Wnt/ $\beta$-catenin-dependent signaling pathway is well-documented, little is known about how growth factors affect $\beta$ - 
catenin-independent (also referred to as non-canonical) Wnt signaling. The $\beta$-cateninindependent Wnt pathway behaves as a double-edged sword; it suppresses tumorigenesis in normal epithelium and in early tumors, but also serves as a critical driver of epithelialmesenchymal transition [EMT] and cancer invasion (17-27). We recently defined a novel paradigm in Wnt signaling in which Frizzled receptors (FZDs) activate the G proteins and trigger non-canonical Wnt signaling via Daple (CCDC88C), which is a Dishevelled (Dvl)binding protein (28). Daple directly binds Wnt5a-activated FZDs, and serves as a guaninenucleotide exchange factor (GEF) that activates the $G$ protein, $G_{a i}$ (Fig. 1A). Upon ligand stimulation, Daple-GEF dissociates from Dvl, binds and displaces Dvl from FZDs (Fig. 1B), and assembles Daple-Gai complexes (Fig. 1C). Disassembly of Daple:Dvl complexes and formation of FZD:Daple:Gai complexes facilitates the activation of trimeric $\mathrm{G}_{a i}$ near ligand-activated FZDs. Activation of $\mathrm{G}_{\mathrm{ai}}$ by Daple-GEF suppresses cAMP, whereas released 'free' $\mathrm{G}_{\beta \gamma}$ heterodimers enhance Rac1 and PI3K-Akt signals (Fig. 1A). We and others have shown that Daple-dependent enhancement of non-canonical Wnt signals can suppress tumor growth (28), but can also fuel EMT, trigger cancer cell migration and invasion $(28,29)$ and drive metastasis (18). Furthermore, elevated expression of Daple-GEF in circulating tumor cells prognosticates a poor outcome (30). In doing so, Daple behaves like a double-edged sword-a tumor suppressor early during oncogenesis, but mimics an oncogene and fuels metastatic invasion later.

We investigated what triggers the dissociation of Daple:Dvl complexes and assembly of Daple:Gai complexes as well as how a tumor suppressor like Daple converts into an oncogene during the course of cancer progression. We found that growth factor-RTKs could trigger both events and hijack the Daple- $G_{a i}$ cascade during cancer progression. Findings also reveal how aberrant growth factor signaling may reprogram a tumor suppressive pathway to potentiate non-canonical Wnt signals that drive EMT during cancer progression.

\section{Results}

\section{Allosteric regulation of Daple's G protein regulatory function by its C-terminal PDZ-binding motif}

We previously reported that upon Wnt5a stimulation, cytosolic Daple:Dvl complexes dissociate, and instead Daple: $\mathrm{G}_{\mathrm{ai} 3}$ complexes assemble (28). In vitro protein-protein interaction assays with purified recombinant proteins had revealed that Daple's ability to bind Dvl or $\mathrm{G}_{a \mathrm{i}}$ is mutually exclusive; binding of the PDZ domain of Dvl to the C-terminal PDZ-binding motif [PBM] in Daple competes with the binding of $\mathrm{G}_{a \mathrm{i}}$ to the $\mathrm{G}_{\mathrm{ai}}$-binding and activating [GBA] module of Daple (Fig. 1C), and an increasing concentration of $\mathrm{G}_{\mathrm{ai}}$ displaces Daple from Dvl (28). Such competition had been unexpected because the GBA [aa 1665-1685] and the PBM [aa 2025-2028] modules in Daple are separated by 350 aa and are within a predicted disordered stretch of the molecule with no semblance to known 3Dstructural modules, suggesting that the observed competition between Dvl :Daple-PBM and $\mathrm{G}_{\mathrm{ai}}$ :Daple-GBA interactions may be allosteric. Here, when we analyzed Dvl :Daple and $\mathrm{G}_{\mathrm{ai}}$ :Daple interactions in Cos7 cells using co-immunoprecipiation assays, we found that binding of Daple to $G_{a i}$ was consistently higher (increased $\sim 2$ fold) when the PBM module of Daple was deleted [Daple- $\triangle$ PBM, a mutant that cannot bind Dvl (31)] (Fig. 1D; Fig. 
S1A). Conversely, binding of Daple to Dvl was consistently higher (increased $\sim 2$ fold) when the GBA module of Daple was disabled by a single point mutation [Daple-F1675A, a mutant that cannot bind $\mathrm{G}_{a i}$; (28)] (Fig. 1E; Fig. S1B). These findings suggest that the PBM and GBA modules of Daple allosterically inhibit each other. Because the increase in binding of Daple- $\triangle P B M$ to $G_{a i 3}$ was also seen in in vitro pulldown assays in which the $G$ protein was bacterially expressed (Fig. S1C-D), we conclude that allosteric inhibition of Daple: $\mathrm{G}_{\mathrm{ai}}$ interaction by Daple's PBM may be due to change in the properties of Daple, not $\mathrm{G}_{\mathrm{ai}}$. Although the structural basis for such inhibition remains unknown, based on these findings and our prior results (28), we conclude that the Dvl-PDZ:Daple-PBM and $\mathrm{G}_{\mathrm{ai}}$ :Daple-GBA interactions antagonistically inhibit each other. Binding of Dvl to Daple may therefore suppress Daple:Gai-dependent enhancement of FZD/non-canonical Wnt signaling. These findings agree with others' observations that overexpression of Dvl can indeed suppresses FZD/G protein-dependent non-canonical Wnt signaling (32). Release of such suppression, which appears to be allosteric, may initiate or enhance Daple-dependent $G$ protein signaling in cells.

\section{Multiple tyrosine kinases phosphorylate Daple's PBM}

We hypothesized that post-translational modifications at the Dvl-PDZ:Daple-PBM interface may disrupt the interface and initiate Daple: $\mathrm{G}_{\mathrm{ai}}$-dependent non-canonical Wnt signaling in cells. We noted that Daple's C-terminal PBM $\left[\underline{Y}^{2023} \mathrm{EY}^{2025} \mathrm{GCV}^{2028}-\mathrm{COOH}\right]$ has two evolutionarily conserved tyrosines (Fig. 2A). Multiple kinase prediction programs (Scansite Motif Scan, MIT; NetPhos 2.0, Denmark; KinasePhos, Taiwan; Phospho-Motif Finder, HPRD) indicated that RTKs and non-RTKs may phosphorylate Daple at those tyrosines. We carried out in vitro kinase assays using various recombinant TKs and wild-type [WT] and non-phosphorylatable mutants of His-tagged purified Daple-CT [aa 1650-2028] protein (in which Tyr, Y2025, either alone (YF), or along with Y2023 (Y2F), is mutated to Phe, F) as substrate. We found that while Y2025 can be phosphorylated by all RTKs tested (EGFR, PDGFR, InsR) as well as the non-RTK Src, Y2023 is phosphorylated exclusively by Src (Fig. 2B-C). MALDI MS/MS spectra of phosphorylated peptides [aa 1650-2028; this segment contains a total of 5 tyrosines] confirmed that while RTKs like EGFR phosphorylated Daple at Y1750 and Y2025, Src phosphorylated Daple at tyrosines Y1655, Y2023 and Y2025; only one of them, Y1699 has been identified as a phosphosite in cells using high-throughput phosphoproteomics (HTP) (www.Phosphosite.org) (Fig. S2B). Y2023 and Y2025 were phosphorylated at $~ 75 \%$ efficacy. Using WT and a non-phosphorylatable mutant of Daple (in which Y2025 is mutated to Phe, F [YF]) and a generic anti-pan-pTyr (pY) antibody for immunoblotting, we confirmed that Y2025 is indeed phosphorylated in cells responding to EGF (Fig. 2D), as well as in cells expressing constitutively active Src (Src-CA; Fig. 2E). Using a combination of Daple-WT, Daple- $\triangle$ PBM [which lacks Y2025], Daple-YF, and another non-phosphorylatable mutant in which both Y2023 and Y2025 were mutated to F [Y2F] we further confirmed that Src indeed phosphorylated Daple at both tyrosines, Y2025 and Y2023 (Fig. 2B-C; Fig. S2E). Finally, we generated an antibody against pY2023 and pY2025 and confirmed that it can detect Daple exclusively upon phosphorylation in vitro (Fig. S2E-F) and in Cos7 cells (Fig. S2G-H). Using this antibody, we further confirmed that endogenous Daple is phosphorylated at Y2023 and Y2025 in HeLa cells responding to EGF (Fig. 2F). We conclude that multiple RTKs and the non-RTK 
Src phosphorylate Daple at Y2025 within the PBM, and that Src can also phosphorylate Daple at Y2023. ROR1 and ROR2, two closely-related TKs that are also activated by Wnt5A and are involved in $\beta$-catenin-independent Wnt signaling (33-35), did not phosphorylate Daple on Y2023/25 either in vitro [using recombinant commercially obtained kinase or tagged overexpressed ROR1/2 immunoprecipitated from Wnt5A-activated HEK cells] or in cellulo [using overexpressed ROR2] (not shown).

\section{Daple is required for activation of $G_{a i}$ and enhancement of Akt and Rac1 signals downstream of EGFR}

Next we asked how the newly discovered phosphoevent on Daple may impact EGF signaling. We hypothesized that if tyrosine phosphorylation triggers a switch of Daplebound complexes from Dvl to $G$ protein, some of the G protein regulatory functions of Daple we reported previously, e.g., activation of $\mathrm{G}_{a \mathrm{i}}$ and enhancement of Akt and Rac1 signals (28) must be impacted in cells responding to growth factors. To answer that, first we asked if Daple and its ability to bind and activate $G_{a i 3}$ was essential for cellular response to EGF, the ligand for the prototype RTK, EGFR. We found that Daple is essential for EGF response in a manner similar to what we reported previously in the case of Wnt5A/FZD7 (28) - First, using an anti- $\mathrm{G}_{a \mathrm{i}}-\mathrm{GTP}$ monoclonal antibody that specifically recognizes $\mathrm{G}_{\mathrm{ai1}-3}$ in a GTP-bound active conformation (36) and previously validated by us to study DapleGEF (28), we found that compared to controls Daple-depleted HeLa cells failed to enhance $\mathrm{G}_{\mathrm{ai3}}$ activity in response to EGF (Fig. 3A; Fig. S3), indicating that Daple is required for such activation. Second, Daple-depleted HeLa cells also failed to enhance Akt phosphorylation (Fig. 3B-C) and Rac1 activity (Fig. 3D-E), the two downstream pathways previously shown to be dependent on the release of 'free' $G_{\beta \gamma}$ from $G_{i \alpha \beta \gamma}$ heterotrimers by Daple-GEF (28).

More specifically, we asked if Daple's ability to activate $G_{a i}$ was necessary for the enhancement of Akt and Rac1 pathways downstream of EGFR. For this we used previously characterized (28) HeLa cells that are depleted of endogenous Daple and stably express either WT or a GEF-deficient Daple-F1675A (FA) mutant at levels close to endogenous levels. Upon EGF stimulation, HeLa-Daple-WT, but not Daple-FA cells enhanced Akt (Fig. 3F-G) and Rac1 (Fig. 3H-I). When it came to markers of EMT, we previously showed that compared to Daple-WT, Daple-FA cells have significantly lower levels of expression at baseline of Vimentin (VIM), Lysyl-oxidase (LOXL3) and the EMT-inducing transcriptional repressor ZEB1 (28). We found that such holds true also for the adhesion molecule, EPCAM (Fig. 3J), which can act as a double-edged sword during metastasis (37). Because none of these markers showed ligand (EGF)-dependent changes above the baseline changes, these findings suggest that reversible regulation of Daple's PBM may not have long term effects on the transcription of the EMT target genes above and beyond the maximal steady-state effects already attributable to the presence or absence of Daple.

Finally, we analyzed the effect of Daple on EGF stimulated changes in canonical Wnt $\rightarrow \beta$ catenin/TCF/LEF target genes. We also found that the expression of several $\beta$ catenin/TCF/LEF target genes were enhanced in Daple-depleted cells (Fig. 3K), indicating that Daple antagonizes the $\beta$-catenin-dependent Wnt pathway in a manner similar to what 
we observed previously downstream of Wnt5A. Taken together, these studies indicate that much like the Wnt5A/FZD pathway (28), Daple and its G protein modulatory function is required for several components of signaling also downstream of the EGF/EGFR pathway. We hypothesized, tyrosine phosphorylation of Daple's PBM may serve as an acute trigger for the Daple $\rightarrow \mathrm{G}_{\mathrm{ai}}$ axis by simply disrupting the Daple:Dvl interaction and releasing the allosteric inhibition imposed by Dvl.

\section{Tyrosine phosphorylation abolishes Daple's ability to bind Dvl}

We next asked if the binding specificity between Dvl-PDZ and Daple-PBM is controlled by the newly identified phosphoevents within/flanking Daple's PBM. We found that binding of His-Daple-CT to GST-Dvl2-PDZ was reduced when the Daple-CT protein was phosphorylated in vitro by EGFR (Fig. 4A; S4A). EGFR-phosphorylated Daple, however,

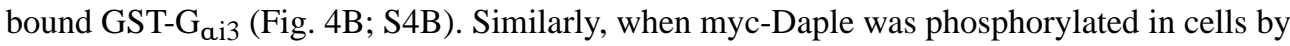
co-expressing the catalytically active wild-type Src kinase, binding of Src-phosphorylated full length Daple to GST-Dvl-PDZ was also reduced (Fig. 4C, E; S4C). By contrast, binding of Src-phosphorylated full-length Daple to GST-G ai3 $_{3}$ was increased (Fig. 4D, E; S4C).

To study the effect of phosphorylation of Daple-PBM in cells, we generated phosphomimicking and non-phosphorylatable mutants of full length myc-Daple in which Y2025, the multi-RTK substrate site was replaced with either glutamate (YE) or phenylalanine (YF), respectively. Additional mutants were created in which both Y2025 and Y2023 were substituted (Y2E, or Y2F) to study the effect of Src-dependent phosphorylation on those residues. We found that both phosphomimicking myc-Daple mutants, YE and Y2E, failed to bind GST-Dvl-PDZ (Fig. 4F; Fig. S4D). Unexpectedly, we observed that the nonphosphorylatable YF mutant of Daple also bound poorly to Dvl ( 60\% less than WT; Fig. 4F; Fig. S4E) suggesting that the residue Y2025 may play an important role in the interface between Daple and Dvl that cannot be fulfilled by Phe [although both Y and F feature a 6carbon aromatic ring, the latter lacks a hydroxyl(-OH) group which imparts its polar characteristics and allows participation in hydrogen $(\mathrm{H})$-bonds and improved protein stability (38)].

To gain structural insights into these findings, we generated a 3D homology-based model of Daple's PBM bound to Dvl's PDZ domain using previously solved structures of Dvl2-PDZ domain co-crystallized with various peptides from the Pocketome (39). The model showed that while both tyrosines are predicted to interact directly with Dvl by forming H-bonds [Y2025 with Asp (D)331 in Dvl, and Y2023 with Ser(S)286 in Dvl], the two tyrosines also stabilize each other with a third H-bond (Fig. 4G). These findings explain why negative charge generated by phosphorylation at Y2025 (or Y2025E mutation) alone is sufficient to abolish Daple:Dvl interaction [likely to repel D331 on Dvl]. Because the interface is enriched in Tyr- $\mathrm{OH}$ mediated H-bonds, replacement of $\mathrm{Y}$ with $\mathrm{F}$ is indeed predicted to destabilize/weaken the Daple-PBM:Dvl-PDZ interface, consistent with findings in our binding assays (Fig. 4A-F). These results are consistent with prior carefully conducted $(\mathrm{Y} \rightarrow \mathrm{F})$ mutagenesis experiments which have documented the importance of $\mathrm{Y}-\mathrm{OH}$ mediated interactions to the energy of protein-protein interactions (40). 
To determine if these findings with in vitro-phosphorylated Daple or phosphomimicking YE mutants hold true in cells, we analyzed the abundance of Dvl:Daple complexes in cells before and after stimulation of the prototype RTK EGFR with EGF. Co-immunoprecipitation studies confirmed that the amount of Daple complexed with Dvl drops significantly (by 75-80\%) within 15 min after EGF stimulation (Fig. 4H; S4F), like what was observed in response to Wnt5a ligand (28). Taken together, these findings demonstrate that growth factors can indeed disrupt the Daple:Dvl interaction, and that tyrosine phosphorylation of Daple's PBM by multiple RTKs and non-RTKs could serve as one such trigger, a mechanism for convergent signaling.

\section{Tyrosine phosphorylation of Daple favors binding to and activation of Gai downstream of EGF}

Next we asked if the loss of Dvl:Daple interaction upon EGF stimulation enhances Daple's ability to bind and activate $\mathrm{G}_{\mathrm{ai}}$. Consistent with our prior findings that tyrosinephosphorylation of Daple favors interaction with G protein (Fig. 4D), binding of Daple to G protein was increased $\sim 2.5$-fold after EGF stimulation (Fig. 4I; S4G), and binding for YE was enhanced $\sim 1.5$ fold and binding of the YF mutant was abolished (Fig. 4J; Fig. S4H). As for binding to FZD7, it appeared that tyrosine phosphorylation may have no effect because both Daple-WT and Daple-YE bound to a similar extent; however, Daple-YF bound less (Fig. S4I-J). Finally, we confirmed that increased binding of $G_{a i}$ to Daple-YE [observed in 4J] indeed translated to increased $\mathrm{G}$ protein activity in cells because compared to cells expressing Daple-WT, activation of $\mathrm{G}_{\mathrm{ai}}$ was higher in cells expressing the phosphomimicking Daple-YE mutant [which mimics constitutive phosphoactivation of Daple by TKs; Fig. 4K-L; S4K]. Thus, the phosphomimicking YE mutant did not bind Dvl, but bound and activated $\mathrm{G}_{\mathrm{ai}}$ more efficiently, and had no effect at all on binding to FZD7. The non-phosphorylatable YF mutant, on the other hand, bound Dvl and FZD7, albeit with reduced affinity, but did not bind $\mathrm{G}_{\mathrm{ai}}$.

We previously showed that activation of $\mathrm{G}_{\mathrm{ai}}$ by Daple downstream of the Wnt5A/FZD7 pathway reduced cellular concentration of the second messenger, cyclic AMP (cAMP). We asked if activation of $\mathrm{G}_{\mathrm{ai}}$ that is initiated by tyrosine phosphorylation of Daple translates into inhibition of cAMP. We found that in control cells [sh-C; Fig. 4M] EGF stimulation leads to a small ( 25-30\% increase) but significant increase in cAMP, but in cells without Daple [sh-Daple] such increase is 500\%, indicating that Daple is required for suppression of cAMP after EGF stimulation. This phenotype was reversed in Daple-depleted cells stably expressing Daple-WT. Basal levels of cAMP in cells expressing the YE and YF mutants were consistent with their $\mathrm{G}_{\mathrm{ai}}$-binding properties (Fig $4 \mathrm{~J}$ ), in that, compared to Daple-WT, the YE mutant had reduced cAMP, whereas the YF mutant had elevated cAMP (Fig 4M). In addition, cells expressing Daple-YE were non-responsive to EGF ligand, in that it showed no ligand-dependent increase in cAMP.

Taken together, these findings indicate that the two tyrosines in Daple's PBM have a direct impact on binding to Dvl, and impacts two other key interactions mediated by distinct modules in Daple-CT that are located upstream of the PBM, i.e., FZD-binding domain and $\mathrm{G}_{\mathrm{ai}}$-binding and activating (GBA) motif (see Fig. S2A). Although the mechanisms of such 
allosteric effect remain unclear, phosphorylation of the two tyrosines in Daple-PBM downstream of growth factor RTKs abolishes Daple's ability to bind Dvl, but increased its ability to bind and activate $\mathrm{G}_{a \mathrm{i}}$ and inhibit cellular cAMP (Fig. 4N).

\section{Tyrosine phosphorylation of Daple's PBM enhances non-canonical Wnt signals, triggers EMT}

To study the impact of tyrosine-phosphorylation of Daple on pathways (Rac1 and PI3K/Akt signals) previously attributed to Daple, we generated HeLa cell lines stably depleted of endogenous Daple and rescued by expressing Daple-WT or various YE/YF mutants at close to endogenous levels (Fig. S5A). Because Y2025 is a common target of RTKs and nonRTKs and substitution of Y2025 with E is sufficient for maximally dissociating Daple:Dvl complexes and for binding and activating $\mathrm{G}_{\mathrm{ai}}$, we proceeded with in-depth characterization of the cells expressing Daple-WT and the YE mutant; the latter mimics a constitutively phosphoactivated state and is sufficient for abolishing the Daple:Dvl interaction (Fig. 4F) and augmenting Daple's ability to bind and activate $\mathrm{G}_{\mathrm{ai}}$ (Fig. 4I-M). Because the nonphosphorylatable YF mutations continued to bind Dvl (Fig. 4F) and FZD7 (Fig. S4D-E), albeit weaker than Daple-WT, but abolished interaction with $\mathrm{G}_{\mathrm{ai} 3}$ (Fig. 4J), we cautiously analyzed this mutant as a non-phosphorylatable control that is likely to be non-responsive to EGF stimulation.

Hyperactivation of $\mathrm{G}_{\mathrm{ai}}$ in Daple-YE cells was associated with the enhancement of all previously published functions of Daple-GEF (28). For example, compared to Daple-WT cells, Daple-YE cells displayed a greater antagonistic suppression of both levels of $\beta$-catenin protein (Fig. 5A-B; Fig. S5B-C) as well as levels of expression of its transcriptional targets (myc, CCND1 and SFRP1; Fig. 5C, left panel). Surprisingly, two other targets of $\beta$-catenin, osteopontin [OPN; which is known as a master regulator of EMT (41) via its ability to stabilize vimentin (42)] and Axin2 [AXIN2, which enhances EMT via induction of Snail (43)] were not suppressed, and instead were increased in Daple-YE cells (Fig. 5C, right panel). Thus, constitutive phosphoactivation of Daple's PBM mimicked in Daple-YE cells was associated with suppression of $\beta$-catenin/TCF/LEF transcriptional targets, but key targets that trigger EMT escaped such suppression. Suppression of $\beta$-catenin-dependent Wnt signaling in Daple-YE cells was accompanied also by reduced growth of these cells under both anchorage-dependent and -independent conditions (Fig. 5D-E; Fig. S5D-G). Compared to Daple-WT cells, Daple-YE cells displayed higher levels of Rac1 activity (Fig. 5F) and Akt phosphorylation (Fig. 5G), enhanced expression of markers of EMT (VIM and LOXL3; Fig. 5H-I), and increased migration, as determined by transwell chemotaxis assays (Fig. 5J). Daple-Y2E cells resembled the phenotypes observed in Daple-YE cells, only enhanced to a greater degree (Fig. S5B-I). Cells expressing Daple-Y2F (a mutant that cannot bind $\mathrm{G}_{\mathrm{ai}}$, and binds poorly to Dvl and FZD) displayed an opposite phenotype; these cells showed increased colony growth and reduced migration (Fig. S5B-J), and in doing so, resembled the previously characterized GEF-deficient Daple-F1675A mutant that cannot bind or activate $\mathrm{G}_{\mathrm{ai}}$ (28). These findings are consistent with other instances in which imbalances in PDZ:PBM interactions perturb cellular homoeostasis and contribute to tumor cell phenotypes that ultimately fuel cancer progression [reviewed in (44)]. 
Selective enhancement of some EMT-associated $\beta$-catenin/TCF/LEF-dependent target genes (OPN and AXIN2) and suppression of other proliferation-associated transcriptional targets in Daple-YE cells (Fig. 5C), despite the relatively lower levels of $\beta$-catenin in these cells (Fig. 5A-B) was an unexpected observation. Such differential response of $\beta$ catenin/TCF/LEF target genes in Daple-YE cells responding to EGF could be due to differences in either the distribution or activity of the remaining pool of $\beta$-catenin. In fact, we found that enhanced Akt phosphorylation in Daple-YE cells is accompanied also by enhanced phosphorylation of $\beta$-catenin at $\operatorname{Ser}(\mathrm{S}) 552$ (Fig. 5G) and its nuclear localization (Fig. S6A-B); phosphorylation by Akt at that site is known to enhance the transcriptional activity of $\beta$-catenin and promote tumor cell invasion (9). It is possible that higher activity of a smaller pool of $\beta$-catenin in Daple-YE cells is sufficient for targets like AXIN2 and OPN which are ultra-responsive to $\beta$-catenin/TCF/LEF (because of the large number of TCF sites in its promoter), but not sufficient for other targets. Additional factors (additional posttranslational modifications, distribution, proteasomal degradation, protein-protein interactions) that affect the functions of $\beta$-catenin may coexist, and cannot be ruled out.

\section{Concurrent upregulation of Daple and EGFR in colorectal tumors carries poor prognosis}

To determine the impact of crosstalk between growth factors and Daple-GEF on clinical outcome, we compared the mRNA expression levels to disease-free survival (DFS) in a data set of 466 patients with colorectal cancers (see Methods). Patients were stratified into negative (low) and positive (high) subgroups with regard to Daple (CCDC88C) and EGFR gene-expression levels with the use of the StepMiner algorithm, implemented within the Hegemon software (hierarchical exploration of gene-expression microarrays online; (45)] (Fig. 6A). Kaplan-Meier analyses of DFS over time showed that among patients with high EGFR, expression of Daple at high levels carried a significantly poorer prognosis compared to those with low Daple (Fig. 6B). Among patients with low EGFR, high expression of Daple was associated with a protective effect, although the trend was not statistically significant (Fig. 6C). Conversely, among patients with high levels of Daple, survival was significantly different between those with high vs low EGFR (Fig. 6D); no such trend was noted among patients with low Daple (Fig. 6E). In fact, the high Daple/high EGFR signature carried a poorer prognosis compared to all other patients combined (Fig. S7). Together, these results indicate that concurrent upregulation of the growth factor and non-canonical Wnt pathways spurred by Daple is associated with poorer clinical outcomes. Findings are in keeping with a working model (see legend Fig 6F) in which G protein signaling via Daple can be triggered by both growth factor and non-canonical Wnt cascades, two pathways that are frequently upregulated during oncogenic progression and are known to promote tumor cell dissemination $(28,46)$.

\section{Discussion}

Previously we showed that non-canonical Wnt signals initiated upon binding of Wnt5a to FZDs are enhanced and propagated by the multimodular non-receptor GEF, Daple, via activation of Gai proteins (28). The major discovery in the current work is an alternate path for activation of Daple-dependent non-canonical Wnt signaling, one that is triggered by growth factors. In both instances, a key mechanistic step is that ligand stimulation [either 
Wnt5a (28) or EGF (current work)] triggered the assembly of Daple: $\mathrm{G}_{\mathrm{ai}}$ complexes to the detriment of Daple:Dvl complexes (Fig. 6F); such a switch in the composition of Daplebound complexes is a pre-requisite for activation of $\mathrm{G}$ protein and enhancement of noncanonical Wnt signals through $\mathrm{G}$ protein intermediates [i.e., cAMP and 'free' $\mathrm{G}_{\beta \gamma}$ ]. Taken together, these results place Daple at the point of convergence between the Wnt/FZD, the growth factor RTKs, and G proteins/GPCRs, three major signaling mechanisms that are conventionally thought to operate independently. In doing so, this work defines a new paradigm in signal transduction, the first one of its kind at the cross-road of non-canonical Wnt and growth factor signaling pathways.

Mechanistically, the convergence between the three major pathways, the so-called cross-talk, plays out at the level of Daple, $G_{a i}$ and Dvl. Our experimental evidence indicates that Dvl and $\mathrm{G}_{\mathrm{ai}}$ compete for binding Daple; such competition appears to be allosteric. By triggering tyrosine phosphorylation of Daple's PBM, growth factors working via activation of multiple TKs facilitate the dissociation of Daple:Dvl complexes and favor the assembly of Daple: $\mathrm{G}_{\mathrm{ai}}$ complexes. This phenomenon has two clear implications-First, it marks another point of convergent signaling in which multiple RTKs and non-RTKs are able to phosphorylate Daple's PBM to initiate Daple $\rightarrow G_{a i}$ cascade. It is noteworthy that convergent signaling is a common phenomenon within this family of proteins; Girdin/GIV, the paralogue of Daple and the prototypical member of this family of GEFs is also phosphorylated by multiple RTKs and non-RTKs at two tyrosines within its C-terminus, resulting in a common outcome —direct binding and activation of class 1 PI3-kinases. Second, Dvl, and perhaps other PDZ proteins that may also bind Daple's PBM, and thereby serve as negative allosteric modulators (NAMs) of the Daple $\rightarrow \mathrm{G}_{\mathrm{ai}}$ cascade. These findings do not contradict the wellaccepted notion that Dvl is required for non-canonical Wnt signaling, or that the Dvl:Daple interaction is required for Daple-dependent Wnt signaling (29). Because Dvl:Daple and Daple: $G_{a i}$ interactions antagonize each other, but both are essential for Daple-dependent non-canonical Wnt signaling cascade, we conclude that-1) Dvl-PDZ may serve as a NAM for Daple-GEF, and 2) reversible dynamic phosphoregulation of the Dvl:Daple interaction may be critical for initiating (when phosphorylated) and terminating (when dephosphorylated) signaling via Daple-GEF.

Our results are also in keeping with the fact that many PDZ:PBM interactions between a variety of signaling molecules are known to be regulated by phosphoevents [reviewed in (44)]. For example, multiple S/T kinases [like Bcr (47), CAMKII (48), PKA (49)] are known to target either PDZs or PBMs. Although tyrosine phosphorylation of PBM has been reported in at least one instance, i.e., Syndecan-1 (SDC1), such phosphorylation did not impact binding to its PDZ partner, Tiam1 (50) [a key distinction from Daple is the position of $\mathrm{Y}$ in SDC1-PBM at next to the last aa from the end]. We conclude that disruption of Daple:Dvl binding by phosphoevents triggered by TKs is the first example of tyr-kinasebased signaling regulating a PDZ:PBM interaction and impacting $G$ protein signaling. These insights pinpoint a clear 'event' that involves players from all three signaling pathways, i.e., TKs, Dvl and $G$ proteins. Findings also add to the crosstalk between heterotrimeric $G$ proteins and Dvl that have been reported previously $-G_{\beta \gamma}$ subunits released from $G_{a i}$ subunits can bind Dvl (51-53), and target it for degradation (54). Further studies are warranted to assess the ramifications of this crosstalk. 
Our findings using phosphomimicking (YE) and non-phosphorylatable (YF) tyrosine Daple mutants in cell-based assays and hierarchical exploration of gene-expression microarrays on patient-derived colorectal tumors shed light onto how concurrent deregulation in growth factor and non-canonical Wnt signaling pathways may impact cancer progression. Although the major physiologic function of non-canonical Wnt signaling is in the establishment of planar cell polarity, tissue morphogenesis and suppression of tumors, non-canonical Wnt signaling is also known to promote the invasiveness and malignant progression of cancers (55); the latter traits are also fuelled by growth factors (56). Overexpression of Wnt5a has been found to be associated with aggressive tumor biology and poor prognosis (57-59). Deregulated growth factor signaling (e.g., copy number variations or activating mutations in RTKs, increased growth factor production/concentration) is also often encountered in advanced tumors (56). We previously showed (28) that Daple enhances non-canonical Wnt signaling via its ability to activate G-proteins downstream of Wnt5a/FZD7; it serves as a tumor suppressor in the normal epithelium and in early tumors (28), but aids metastatic progression in advanced tumors and in circulating tumor cells $(18,28,30)$. What triggers such role-reversal, was unknown. We propose that when Wnt and growth factor pathways are deregulated concurrently during cancer progression [likely due to sequential mutations within each pathway, first in Wnt followed by growth factors], growth factor RTKs phosphorylate Daple's PBM and augment the prometastatic Daple-dependent non-canonical Wnt signals, thereby fueling cancer dissemination.

In conclusion, we have provided evidence for how the multimodular signal transducer, Daple, provides a platform for complex crosstalk between non-canonical Wnt, growth factor RTKs, and G protein signaling cascades in multi-receptor driven diseases such as cancers. Findings also illuminate how such crosstalk coordinately shapes cellular phenotypes, sometimes even by reprogramming tumor suppressive pathways to aid tumor dissemination.

\section{Materials and Methods}

\section{Reagents and Antibodies}

Unless otherwise indicated, all reagents were of analytical grade and obtained from SigmaAldrich (St. Louis, MO). Cell culture media were purchased from Invitrogen (Grand Island, NY). All restriction endonucleases and Escherichia coli strain DH5a were purchased from New England Biolabs (Ipswich, MA). E. coli strain BL21 (DE3), phalloidin-Texas Red were purchased from Invitrogen. Genejuice transfection reagent was from Novagen (Madison, WI). PfuUltra DNA polymerase was purchased from Stratagene (La Jolla, CA). Goat antirabbit and goat anti-mouse Alexa Fluor 680 or IRDye $800 \mathrm{~F}\left(\mathrm{ab}^{\prime}\right) 2$ used for immunoblotting were from Li-Cor Biosciences (Lincoln, NE). Mouse anti-His, anti-a tubulin and anti-actin were obtained from Sigma; anti-Myc and anti-HA were obtained from Cell Signaling Technology (Beverly, MA) and Covance (Princeton, NJ), respectively. Mouse monoclonal antibody against phosphotyrosine (pTyr, Cat \# 610000) was obtained from BD Transduction Laboratories (San Jose, CA). Rabbit anti-pan-G $\beta$ (M-14), anti-G $\mathrm{ai}_{\mathrm{a} 3}$, anti-Dvl and anti- $\beta$ catenin were obtained from Santa Cruz Biotechnology (Dallas, TX); anti-phospho-Akt (S473) and anti-pan Akt were obtained from Cell Signaling Technology; anti-Rac1 was obtained from BD Transduction Laboratories. Rabbit polyclonal anti-Daple (total) antibody 
were generated in collaboration with Millipore (Carlsbad, CA) using the C-terminus of Daple (aa 1660-2028) as an immunogen and validated previously (28).

An affinity purified rabbit polyclonal phospho-specific anti-Daple (pY2023, pY2025) antibody was generated using a phosphopeptide corresponding to the PBM motif in human Daple as immunogen by $21^{\text {st }}$ Century Biochemicals (Marlboro, MA). Briefly, rabbits were immunized with a 1:1 mix of 2 phosphopeptides-Ahx-PQTVWYE[pY]GCV-OH and AhxPQTVW[pY]E[pY]GCV-OH, and purified by adsorbing antibodies against the nonphosphorylated sequence using a Ahx-PQTVWYEYGCV-OH peptide.

\section{Plasmid Constructs and Mutagenesis}

Cloning of N-terminally tagged myc-Daple was carried out as described previously (Aznar et al, 2015). All subsequent site-directed mutagenesis and truncated constructs (myc-Daple full length F1675A (FA), myc-Daple deleted from aa 2025-2028 ( $\triangle \mathrm{PBM}$ ), myc-Daple FA $+\Delta$ PBM (2M), myc-Daple Y2025E (YE), Y2023-2025E (Y2E), Y2025F (YF), and Y2023-2025F (Y2F) were carried out using myc-Daple-WT as template using Quick Change as per manufacturer's protocol. The His-Daple-CT WT, YF and Y2F constructs (1650-2028 aa) used for in vitro protein-protein interaction assays were cloned from mycDaple pcDNA 3.1 and inserted within the pGEX-4T vector, respectively, between Ndell EcoRI restriction sites.

Cloning of rat Ga-proteins into pGEX-4T-1 (GST-G $\mathrm{ai}_{3}$ and $\left.\mathrm{G}_{\mathrm{ai} 3}-\mathrm{HA}\right)$ have been described previously (60-64). C-terminal HA-tagged c-Src for mammalian expression was generated by cloning the entire coding sequence into pcDNA 3.1 between XhoI and EcoRI. HA-Src Y527F (constitutively active; CA) and HA-Src K295R (kinase dead; KD) were generated by site-directed mutagenesis using a Quick Change kit (Stratagene, San Diego, CA) as per manufacturer's protocol. All HA-Src constructs have been previously validated by us (65). Several constructs used in this work were generous gifts from other investigators: Untagged-EGFR was from Marilyn G. Farquhar (UCSD, La Jolla,CA) (62); mouse Dvl1 was from Mikhail V. Semenov (Harvard Medical School, Boston, MA); GST-Dvl2-PDZ was from Raymond Habas (Temple University, Philadelphia, PA); GST-tagged FZD7-CT construct (66) was from Ryoji Yao (Japanese Foundation of Cancer Research institute, Japan); GST-PBD (PDZ-Binding Domain) was from Gary Bokoch (The Scripps Research Institute, La Jolla, CA). All these constructs have been previously used and validated by us in our recently published work on Daple (28).

Daple shRNA constructs have been validated and are described in detail elsewhere (28).

\section{Protein Expression and Purification}

GST and His-tagged recombinant proteins were expressed in E. coli strain BL21 (DE3) (Invitrogen) and purified as described previously $(62,63,67)$. Briefly, bacterial cultures were induced overnight at $25^{\circ} \mathrm{C}$ with $1 \mathrm{mM}$ isopropylß-D-1-thio-galactopyranoside (IPTG). Pelleted bacteria from $1 \mathrm{~L}$ of culture were resuspended in $20 \mathrm{~mL}$ GST-lysis buffer $[25 \mathrm{mM}$ Tris· $\mathrm{HCl}, \mathrm{pH}$ 7.5, $20 \mathrm{mM} \mathrm{NaCl}, 1 \mathrm{mM}$ EDTA, 20\% (vol/vol) glycerol, 1\% (vol/vol) Triton $\mathrm{X}-100,2 \times$ protease inhibitor mixture (Complete EDTA-free; Roche Diagnostics)] or in $20 \mathrm{~mL}$ His-lysis buffer [50 mM NaH2PO4 (pH 7.4), $300 \mathrm{mM} \mathrm{NaCl}, 10 \mathrm{mM}$ imidazole, 1\% (vol/ 
vol)Triton X-100, 2xprotease inhibitor mixture (Complete EDTA-free; Roche Diagnostics)] for GST or His-fused proteins, respectively. After sonication (three cycles, with pulses lasting $30 \mathrm{~s} / \mathrm{cycle}$, and with $2 \mathrm{~min}$ interval between cycles to prevent heating), lysates were centrifuged at $12,000 \times$ gat $4{ }^{\circ} \mathrm{C}$ for $20 \mathrm{~min}$. Except for GST-FZD7-CT and GST-PBD constructs (see in vitro GST pulldown assay section), solubilized proteins were affinity purified on glutathione-Sepharose 4B beads (GE Healthcare), dialyzed overnight against PBS, and stored at $-80{ }^{\circ} \mathrm{C}$.

\section{Transfection; Generation of Stable Cell Lines and Cell Lysis}

Transfection was carried out using either PEI as previously described (68) or Genejuice from Novagen for DNA plasmids following the manufacturers' protocols. HeLa cell lines stably expressing Daple constructs were selected after transfection and in the presence of

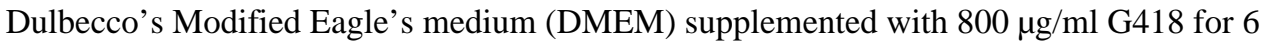
weeks. The resultant multiclonal pool was subsequently maintained in the presence of 500 $\mu \mathrm{g} / \mathrm{ml} \mathrm{G} 418$. Unless otherwise indicated, for assays involving serum starvation, serum concentration was reduced to $0.2 \%$ Fetal Bovine Serum (FBS) overnight for HeLa cells and $0 \%$ FBS for Cos7.

Whole-cell lysates were prepared after washing cells with PBS prior to resuspending and boiling them in sample buffer. Cold PBS $\left[4^{\circ} \mathrm{C}\right]$ was used whenever ligand stimulation was carried out for designated time periods. Lysates used as a source of proteins in immunoprecipitation or pull-down assays were prepared by resuspending cells in Tx-100 lysis buffer [20 mM HEPES, pH 7.2, $5 \mathrm{mM} \mathrm{Mg-acetate,} 125 \mathrm{mM} \mathrm{K}$-acetate, $0.4 \%$ Triton $\mathrm{X}-100,1 \mathrm{mM}$ DTT, supplemented with sodium orthovanadate $(500 \mu \mathrm{M})$, phosphatase from Sigma-Aldrich and protease from Roche Life Science (Indianapolis, IN) inhibitor cocktails], after which they were passed through a $28 \mathrm{G}$ needle at $4^{\circ} \mathrm{C}$, and cleared by centrifuging $(10,000 \times \mathrm{g}$ for $10 \mathrm{~min})$ before use in subsequent experiments.

\section{Quantitative Immunoblotting}

For immunoblotting, protein samples were separated by SDS-PAGE and transferred to PVDF membranes (Millipore). Membranes were blocked with PBS supplemented with 5\% nonfat milk (or with 5\% BSA when probing for phosphorylated proteins) before incubation with primary antibodies. Infrared imaging with two-color detection and band densitometry quantifications were performed using a Li-Cor Odyssey imaging system exactly as done previously (28). All Odyssey images were processed using Image J software (NIH) and assembled into figure panels using Photoshop and Illustrator software (Adobe).

\section{In vitro GST pulldown and Immunoprecipitation Assays}

Purified GST-G $\mathrm{ai}_{\mathrm{ai}}$, GST-DVL-PDZ or GST alone $(5 \mu \mathrm{g})$ were immobilized on glutathioneSepharose beads and incubated with binding buffer [50 mM Tris- $\mathrm{HCl}$ (pH 7.4), $100 \mathrm{mM}$ $\mathrm{NaCl}, 0.4 \%$ (v:v) Nonidet P-40, $10 \mathrm{mM} \mathrm{MgCl} 2,5 \mathrm{mM}$ EDTA, $30 \mu \mathrm{M}$ GDP, 2 mM DTT, protease inhibitor mixture] for $90 \mathrm{~min}$ at room temperature as described before $(62,63,65$, 67). Lysates $(\sim 250 \mu \mathrm{g})$ of Cos7 cells expressing appropriate myc-Daple constructs or purified His-Daple-CT (aa 1650-2028) protein ( $3 \mu \mathrm{g}$ ) were added to each tube, and binding reactions were carried out for $4 \mathrm{~h}$ at $4{ }^{\circ} \mathrm{C}$ with constant tumbling in binding buffer [50 mM 
Tris- $\mathrm{HCl}$ (pH 7.4), $100 \mathrm{mM} \mathrm{NaCl}, 0.4 \%$ (v:v) Nonidet P-40, $10 \mathrm{mM} \mathrm{MgCl}_{2}, 5$ mM EDTA, $30 \mu \mathrm{M}$ GDP, $2 \mathrm{mM}$ DTT]. Beads were washed (4x) with $1 \mathrm{~mL}$ of wash buffer [4.3 mM $\mathrm{Na}_{2} \mathrm{HPO}_{4}, 1.4 \mathrm{mM} \mathrm{KH} \mathrm{PO}_{4}$ (pH 7.4), $137 \mathrm{mM} \mathrm{NaCl}, 2.7 \mathrm{mM} \mathrm{KCl}, 0.1 \%$ (v:v) Tween 20, $10 \mathrm{mM} \mathrm{MgCl} 2,5 \mathrm{mM}$ EDTA, $30 \mu \mathrm{M}$ GDP, $2 \mathrm{mM}$ DTT] and boiled in Laemmli's sample buffer. Immunoblot quantification was performed by infrared imaging following the manufacturer's protocols using an Odyssey imaging system (Li-Cor Biosciences).

GST-FZD7-CT and GST-PBD constructs were immobilized on glutathione-Sepharose beads directly from bacterial lysates by overnight incubation at $4^{\circ} \mathrm{C}$ with constant tumbling. Next morning, GST-FZD7-CT or GST-PBD immobilized on glutathione beads were washed and subsequently incubated with cell lysates at $4^{\circ} \mathrm{C}$ with constant tumbling. Washes and immunoblotting were performed as previously (28).

For immunoprecipitation, cell lysates ( 1-2 $\mathrm{mg}$ of protein) were incubated for $4 \mathrm{~h}$ at $4{ }^{\circ} \mathrm{C}$ with $2 \mu \mathrm{g}$ of appropriate antibody, anti-HA mAb (Covance) for $\mathrm{HA}_{-} \mathrm{G}_{\mathrm{ai} 3}$, anti-Myc (from Cell Signaling) mAb for myc-Daple, anti-Dvl mAb (from Santa Cruz Biotechnology) or their respective pre-immune control IgGs. Protein G (for all mAbs) Sepharose beads (GE Healthcare) were added and incubated at $4^{\circ} \mathrm{C}$ for an additional $60 \mathrm{~min}$. Beads were washed in PBS-T buffer [4.3 mM Na $2 \mathrm{HPO}_{4}, 1.4 \mathrm{mM} \mathrm{KH}_{2} \mathrm{PO}_{4}, \mathrm{pH} 7.4,137 \mathrm{mM} \mathrm{NaCl}, 2.7 \mathrm{mM} \mathrm{KCl}$, $0.1 \%$ (v:v) Tween 20, $10 \mathrm{mM} \mathrm{MgCl}_{2}, 5 \mathrm{mM}$ EDTA, $2 \mathrm{mM}$ DTT, $0.5 \mathrm{mM}$ sodium orthovanadate] and bound proteins were eluted by boiling in Laemmli's sample buffer.

\section{In vitro and in-cellulo Kinase Assays}

In vitro kinase assays were performed using bacterially expressed His $(6 \times$ His, hexahistidine) tagged Daple-CT (His-Daple-CT, aa 1650-2028) proteins ( $\sim$ g ger reaction), and $\sim 50 \mathrm{ng}$ recombinant kinases which were obtained commercially (SignalChem, Canada). Reactions were started by adding $\sim 1000 \mu \mathrm{M}$ ATP and carried out at $25^{\circ} \mathrm{C}$ for 30 min in tyrosine kinase buffer [60 mM HEPES pH 7.5, $5 \mathrm{mM} \mathrm{MgCl}_{2}, 5 \mathrm{mM} \mathrm{MnCl}_{2}, 3 \mu \mathrm{M}$ sodium orthovanadate]. Reactions were stopped by addition of Laemmli sample buffer and boiling at $100^{\circ} \mathrm{C}$.

For cellular phosphorylation assays using overexpressed Daple, Myc-tagged Daple was coexpressed with untagged EGFR or HA-tagged Src-WT, HA-tagged Src-kinase-dead [KD] or HA-tagged Src-constitutively active [CA]. To determine if EGFR phosphorylates Daple, cells were serum starved for 12-16 h, and stimulated with EGF (50 nM) for $10 \mathrm{~min}$. Cells were preincubated for $1 \mathrm{~h}$ with Src inhibitor, PP2 [Calbiochem] as done previously (65). Reactions were stopped using PBS chilled at $4^{\circ} \mathrm{C}$, supplemented with $200 \mu \mathrm{M}$ sodium orthovanadate, and immediately scraped and lysed for immunoprecipitation.

Phosphoproteins in both in vitro and in cellulo assays were visualized by immunoblotting with either a pan-pTyr antibody [BD Biosciences] or a phosphospecific anti-pTyr 2023-2025-Daple rabbit polyclonal antibody [21 ${ }^{\text {st }}$ Century Biochemicals].

\section{Mass Spectrometry}

These studies were carried out as described previously $(69,70)$. The key steps are summarized below. 
Sample preparation-His-Daple-CT proteins used in various in vitro kinase assays were diluted in TNE (50 mM Tris, pH 8.0, $100 \mathrm{mM} \mathrm{NaCl}, 1 \mathrm{mM}$ EDTA) buffer. RapiGest SF reagent (Waters Corp.) was added to the mix to a final concentration of $0.1 \%$ and samples were boiled for $5 \mathrm{~min}$. TCEP (Tris (2-carboxyethyl) phosphine) was added to $1 \mathrm{mM}$ (final concentration) and the samples were incubated at $37^{\circ} \mathrm{C}$ for $30 \mathrm{~min}$. Subsequently, the samples were carboxymethylated with $0.5 \mathrm{mg} / \mathrm{ml}$ of iodoacetamide for $30 \mathrm{~min}$ at $37^{\circ} \mathrm{C}$ followed by neutralization with $2 \mathrm{mM}$ TCEP (final concentration). Proteins samples prepared as above were digested with trypsin (trypsin:protein ratio - 1:50) overnight at $37^{\circ} \mathrm{C}$. RapiGest was degraded and removed by treating the samples with $250 \mathrm{mM} \mathrm{HCl}$ at $37^{\circ} \mathrm{C}$ for $1 \mathrm{~h}$ followed by centrifugation at $14000 \mathrm{rpm}$ for $30 \mathrm{~min}$ at $4^{\circ} \mathrm{C}$. The soluble fraction was then added to a new tube and the peptides were extracted and desalted using $\mathrm{C} 18$ desalting columns (Thermo Scientific, PI-87782).

LC-MS-MS-Trypsin-digested peptides were analyzed by ultra high pressure liquid chromatography (UPLC) coupled with tandem mass spectroscopy (LC-MS/MS) using nanospray ionization. The nano-spray ionization experiments were performed using a TripleTof 5600 hybrid mass spectrometer (ABSCIEX) interfaced with nano-scale reversed-phase UPLC (Waters corporation nano ACQUITY) using a $20 \mathrm{~cm}$-75-micron ID glass capillary packed with 2.5- $\mu \mathrm{m} \mathrm{C18} \mathrm{(130)} \mathrm{CSH}^{\mathrm{TM}}$ beads (Waters corporation). Peptides were eluted from the $\mathrm{C} 18$ column into the mass spectrometer using a linear gradient (5-80\%) of ACN (Acetonitrile) at a flow rate of $250 \mu \mathrm{l} / \mathrm{min}$ for $1 \mathrm{~h}$. The buffers used to create the ACN gradient were: Buffer A $\left(98 \% \mathrm{H}_{2} \mathrm{O}, 2 \% \mathrm{ACN}, 0.1 \%\right.$ formic acid, and $\left.0.005 \% \mathrm{TFA}\right)$ and Buffer B (100\% ACN, 0.1\% formic acid, and $0.005 \%$ TFA). MS/MS data were acquired in a data-dependent way the MS1 data was acquired for $250 \mathrm{~ms}$ at m/z of 400 to $1250 \mathrm{Da}$ and the MS/MS data was acquired from $\mathrm{m} / \mathrm{z}$ of 50 to 2,000 $\mathrm{Da}$. The Independent data acquisition (IDA) parameters were as follows; MS1-TOF acquisition time of 250 milliseconds, followed by 50 MS2 events of 48 milliseconds acquisition time for each event. The threshold to trigger MS2 event was set to 150 counts when the ion had the charge state $+2,+3$ and +4 . The ion exclusion time was set to 4 seconds. Finally, the collected data were analyzed using Protein Pilot 4.5 (ABSCIEX) for peptide identifications.

\section{Gai activity as determined by conformational anti-Gai-GTP mAb}

These assays were carried out exactly as done previously $(28,71)$. Cells were maintained overnight at steady-state in a media containing 0.2\% FBS [if an EGF stimulation was performed] or maintained at steady-state in media containing $10 \%$ FBS prior to lysis. For immunoprecipitation of active Gai3, freshly prepared cell lysates (2-4 mg) were incubated for $30 \mathrm{~min}$ at $4^{\circ} \mathrm{C}$ with the conformational Gai:GTP mouse antibody $(1 \mu \mathrm{g})(36)$ or with control mouse IgG. Protein G Sepharose beads from GE Healthcare (Pittsburgh, PA) were added and incubated at $4^{\circ} \mathrm{C}$ for additional $30 \mathrm{~min}$ (total duration of assay is $1 \mathrm{~h}$ ). Beads were immediately washed 3 times using $1 \mathrm{ml}$ of lysis buffer (composition exactly as above; no nucleotides added) and immune complexes were eluted by boiling in SDS as previously described $(28,71)$. 


\section{Measurement of cyclic AMP}

HeLa cells were serum starved ( $0.2 \% \mathrm{FBS}, 16 \mathrm{~h})$ and incubated with forskolin and isobutylmethylxanthine (IBMX, $200 \mu \mathrm{M}, 20 \mathrm{~min}$ ) followed by EGF (30 min). Reactions were terminated by aspiration of media and addition of $150 \mu \mathrm{l}$ of ice-cold TCA $7.5 \%$ (w/v). cAMP content in TCA extracts was determined by radioimmunoassay (RIA) and normalized to protein [(determined using a dye binding protein assay (Bio-Rad)] exactly as we have done previously (28). Data is expressed as fmol cAMP/ $\mu \mathrm{g}$ total protein.

\section{Measurement of Rac1 activity}

These assays were carried out exactly as done previously (28) with slight modifications in FBS concentration. Briefly, to analyze the role of phosphotyrosine Daple in the regulation of Rac1 activity we used Daple-depleted HeLa cell lines stably expressing Daple-WT or tyrosine mutants. Cells were maintained overnight at steady-state in a media containing $10 \%$ FBS prior to lysis. Lysis was carried out first in RIPA buffer [20 mM HEPES pH 7.4, 180 $\mathrm{mM} \mathrm{NaCl}, 1 \mathrm{mM}$ EDTA, $1 \%$ Triton X-100, 0.5\% sodium deoxycholate, $0.1 \%$ SDS, supplemented with $1 \mathrm{mM}$ DTT, sodium orthovanadate $(500 \mu \mathrm{M})$, phosphatase (Sigma), and protease (Roche) inhibitor mixtures] for $15 \mathrm{~min}$ on ice, and then for an additional $15 \mathrm{~min}$ after addition of an equal volume of Triton X-100 lysis buffer [20 mM Hepes (pH 7.2), 5 mM Mg-acetate, 125 mM K-acetate, 0.4\% Triton X-100, 1 mM DTT, supplemented with sodium orthovanadate $(500 \mu \mathrm{M})$, phosphatase (Sigma), and protease (Roche) inhibitor mixtures]. During the second 15 min of incubation, cells were broken by passing through a 28 -gauge needle at $4{ }^{\circ} \mathrm{C}$ and lysates were subsequently cleared (10,000×g for $\left.10 \mathrm{~min}\right)$ before use. Equal aliquots of lysates were incubated with bead-bound GST-PBD for $1 \mathrm{~h}$ at $4^{\circ} \mathrm{C}$ with constant tumbling. Beads were washed in PBS-T buffer [4.3 $\mathrm{mM} \mathrm{Na}_{2} \mathrm{HPO}_{4}, 1.4 \mathrm{mM}$ $\mathrm{KH}_{2} \mathrm{PO}_{4}, \mathrm{pH} 7.4,137 \mathrm{mM} \mathrm{NaCl}, 2.7 \mathrm{mM} \mathrm{KCl}, 0.1 \%$ (v:v) Tween 20, $10 \mathrm{mM} \mathrm{MgCl} 2,5 \mathrm{mM}$ EDTA, $2 \mathrm{mM}$ DTT, $0.5 \mathrm{mM}$ sodium orthovanadate] and bound proteins were eluted by boiling in Laemmli's sample buffer.

\section{Transwell Cell Migration}

Chemotactic cell migration assays were performed using Corning Transwell plates according to the manufacturer's protocol exactly as done previously (28) with slight modifications in FBS concentration. HeLa cells were trypsinized, counted, and placed in a Transwell with media containing no FBS (75000 cells/well). Media in the bottom chamber of each well were supplemented with $10 \%$ FBS to trigger chemotactic migration. Cells were allowed to migrate for $24 \mathrm{hr}$ and fixed prior to staining. Cells that had successfully migrated to the side of the permeable membrane facing the bottom chamber were visualized by staining the membrane with crystal violet. Cell migration (expressed as number of cells/ high-power field) was quantified by analyzing 15-20 random fields per membrane insert per condition for the number of Giemsa stained cells. Each experiment was repeated 3 times [biological repeats]; 3 technical repeats were included during each biological repeat.

\section{D modeling of Dvl2-PDZ domain bound to the PDZ-binding motif (PBM) of Daple}

The approximate model of the complex was built using the ICM homology modeling platform (72). Structures of Dvl2-PDZ domain co-crystallized with various peptides were 
collected from the Pocketome (39). A structural alignment of the bound peptides was built, and the PDM of Daple was aligned onto it, suggesting the highest sequence homology with the C1 peptide [PDB 3cbx (73)]. The initial model of the DVL2-PDZ: Daple-PBM complex was built by assigning the backbone coordinates of both target molecules to their counterparts in the template (PDB 3cbx). The model was further refined via conformational sampling of peptide and PDB domain residue side chains in internal coordinates, followed by full-atom local backbone minimization in the presence of harmonic distance restraints maintaining the secondary structure of the complex.

\section{Anchorage-dependent tumor growth assay}

Anchorage-dependent growth was monitored on solid (plastic) surface as performed by us previously (28) with slight modifications in FBS concentration. Briefly, approximately $\sim 1000 \mathrm{HeLa}$ cells stably expressing various Daple constructs were plated in 6-well plates and incubated in $5 \% \mathrm{CO}_{2}$ at $37^{\circ} \mathrm{C}$ for $\sim 2$ weeks in $10 \% \mathrm{FBS}$ growth media. Colonies were then stained with $0.005 \%$ crystal violet for $1 \mathrm{~h}$. Each experiment was repeated 3 times [biological repeats]; 3 technical repeats were included during each biological repeat.

\section{Anchorage-independent tumor growth assay}

Anchorage-independent growth of HeLa cells was analyzed in agar as performed by us previously (28) with slight modifications in FBS concentration. Briefly, petri plates $(60 \mathrm{~mm})$ were pre-layered with $3 \mathrm{ml} \mathrm{1 \%}$ Bacto agar (Life Technologies) in DMEM containing 10\% FBS. Approximately $\sim 5000 \mathrm{HeLa}$ cells stably expressing various Daple constructs were then plated on top in $3 \mathrm{ml}$ of $0.3 \%$ agar-DMEM with $10 \%$ FBS. All assays were carried out using three replicate plates at a seeding density of $\sim 5000$ cells/plate. Following overnight incubation in $5 \% \mathrm{CO}_{2}$ incubator, $1 \mathrm{ml}$ DMEM supplemented with $10 \% \mathrm{FBS}$ was added to maintain hydration. After 2 weeks of growth, colonies were stained with $0.005 \%$ crystal violet/methanol for $1 \mathrm{~h}$ and subsequently photographed by light microscopy. The number of colonies in 15-20 randomly-selected fields was counted under 10x magnification. Each experiment was repeated 3 times [biological repeats]; 3 technical repeats were included during each biological repeat.

\section{RNA isolation and qPCR}

These assays were carried out as described previously (28). Total RNA was isolated using an RNeasy kit (QIAGEN) as per the manufacturers' protocol. First-strand cDNA was synthesized using Superscript II reverse transcriptase (Invitrogen), followed by ribonuclease $\mathrm{H}$ treatment (Invitrogen) prior to performing quantitative real-time PCR. Reactions omitting reverse transcriptase were performed in each experiment as negative controls. Reactions were then run on a real-time PCR system (ABI StepOnePlus; Applied Biosystems). Gene expression was detected with SYBR green (Invitrogen), and relative gene expression was determined by normalizing to GAPDH using the $\Delta \mathrm{C}_{\mathrm{T}}$ method. Primer sequences are available upon request. 


\section{Stratification of colon cancer patients in distinct gene-expression subgroups and comparative analysis of their survival outcomes}

The association between the levels of Daple (CCDC88C) and EGFR mRNA expression and patient survival was tested in cohort of 466 patients where each tumor had been annotated with the disease-free survival (DFS) information of the corresponding patient. This cohort included gene expression data from four publicly available NCBI-GEO data-series (GSE14333, GSE17538, GSE31595, GSE37892)(74-77), and contained information on 466 unique primary colon carcinoma samples, collected from patients at various clinical stages (AJCC Stage I-IV/Duke's Stage A-D) by five independent institutions: 1) the H. Lee Moffit Cancer Center in Tampa, Florida, USA; 2) the Vanderbilt Medical Center in Nashville, Tennessee, USA; 3 ) the Royal Melbourne Hospital in Melbourne, Australia; 4) the Institut PaoliCalmette in Marseille, France; 5) the Roskilde Hospital in Copenhagen, Denmark. All 466 samples contained in this subset were cross-checked to exclude the presence of redundancies/duplicates. A complete list of all GSMIDs of the experiments contained within the NCBI-GEO discovery dataset has been published previously (78). To investigate the relationship between the mRNA expression levels of selected genes (i.e. CCDCDDC, Wnt5a, EGFR and FZD7) and the clinical outcomes of the 466 colon cancer patients represented within the NCBI-GEO discovery dataset, we applied the Hegemon, "hierarchical exploration of gene expression microarrays on-line" tool (45). The Hegemon software is an upgrade of the BooleanNet software (79), where individual gene-expression arrays, after having been plotted on a two-axis chart based on the expression levels of any two given genes, can be stratified using the StepMiner algorithm and automatically compared for survival outcomes using Kaplan-Meier curves and log-rank tests. Since all 466 samples contained in the dataset had been analyzed using the Affymetrix HG-U133 Plus 2.0 platform (GPL570), the threshold gene-expression levels for Daple/CCDC88C and EGFR were calculated using the StepMiner algorithm based on the expression distribution of the 25,955 experiments performed on the Affymetrix HG-U133 Plus 2.0 platform. We stratified the patient population of the NCBI-GEO discovery dataset in different gene-expression subgroups, based on either the mRNA expression levels of Daple/CCDC88C alone (i.e. CCDC88C neg vs. pos), EGFR alone (i.e. EGFR neg vs. pos), or a combination of both (i.e. CCDC88Cneg/EGFRpos vs. CCDC88Cpos/EGFRpos vs. CCDC88Cpos/EGFRneg vs CCDC88Cpos/EGFRpos). Once grouped based on their gene-expression levels, patient subsets were compared for survival outcomes using both Kaplan-Meier survival curves and multivariate analysis based on the Cox proportional hazards method.

\section{Statistical analysis}

Each experiment presented in the figures is representative of at least three independent experiments. Displayed images and immunoblots are representative of the biological repeats. Statistical significance between the differences of means was calculated by an unpaired student's t-test or one-way ANOVA [whenever more than two groups were compared]. A two-tailed $p$ value of $<0.05$ at $95 \%$ confidence interval is considered statistically significant. All graphical data presented were prepared using GraphPad or Matlab. 


\section{Supplementary Material}

Refer to Web version on PubMed Central for supplementary material.

\section{Acknowledgments}

We thank Gordon N. Gill and Marilyn G. Farquhar (UCSD) and Deepali Bhandari (CSULB) for their critical input during the preparation of the manuscript.

\section{FUNDING}

This work was supported by NIH grants CA100768, CA160911 and DK099226 (to P.G). P.G. was also supported by the American Cancer Society (ACS-IRG 70-002) and by the UC San Diego Moores Cancer Center. I.K. was supported by NIH grants GM071872, AI118985, and GM117424. I.L-S was supported by a fellowship from the American Heart Association (AHA \#14POST20050025). F.H was supported by the State Scholarship Fund of China Scholarship Council (No. 201208510048) and fund from West China Hospital, Sichuan University, PR China, during her tenure as a visiting professor to UCSD.

\section{References and Notes}

1. Morris SL, Huang S. Crosstalk of the Wnt/beta-catenin pathway with other pathways in cancer cells. Genes Dis. 2016; 3:41-47. [PubMed: 27081668]

2. Anastas JN. Functional Crosstalk Between WNT Signaling and Tyrosine Kinase Signaling in Cancer. Semin Oncol. 2015; 42:820-831. [PubMed: 26615128]

3. Rota LM, Wood TL. Crosstalk of the Insulin-Like Growth Factor Receptor with the Wnt Signaling Pathway in Breast Cancer. Front Endocrinol (Lausanne). 2015; 6:92. [PubMed: 26106366]

4. Peng X, et al. FOXQ1 mediates the crosstalk between TGF-beta and Wnt signaling pathways in the progression of colorectal cancer. Cancer Biol Ther. 2015; 16:1099-1109. [PubMed: 25955104]

5. Song L, Li ZY, Liu WP, Zhao MR. Crosstalk between Wnt/beta-catenin and Hedgehog/Gli signaling pathways in colon cancer and implications for therapy. Cancer Biol Ther. 2015; 16:1-7. [PubMed: 25692617]

6. Bertrand FE, Angus CW, Partis WJ, Sigounas G. Developmental pathways in colon cancer: crosstalk between WNT, BMP, Hedgehog and Notch. Cell Cycle. 2012; 11:4344-4351. [PubMed: 23032367]

7. Yanai K, et al. Crosstalk of hedgehog and Wnt pathways in gastric cancer. Cancer Lett. 2008; 263:145-156. [PubMed: 18243529]

8. Nelson WJ, Nusse R. Convergence of Wnt, beta-catenin, and cadherin pathways. Science. 2004; 303:1483-1487. [PubMed: 15001769]

9. Fang D, et al. Phosphorylation of beta-catenin by AKT promotes beta-catenin transcriptional activity. The Journal of biological chemistry. 2007; 282:11221-11229. [PubMed: 17287208]

10. Ding Q, et al. Erk associates with and primes GSK-3beta for its inactivation resulting in upregulation of beta-catenin. Mol Cell. 2005; 19:159-170. [PubMed: 16039586]

11. Kim SE, Choi KY. EGF receptor is involved in WNT3a-mediated proliferation and motility of NIH3T3 cells via ERK pathway activation. Cell Signal. 2007; 19:1554-1564. [PubMed: 17374561]

12. Yun MS, Kim SE, Jeon SH, Lee JS, Choi KY. Both ERK and Wnt/beta-catenin pathways are involved in Wnt3a-induced proliferation. J Cell Sci. 2005; 118:313-322. [PubMed: 15615777]

13. Lemieux E, Cagnol S, Beaudry K, Carrier J, Rivard N. Oncogenic KRAS signalling promotes the Wnt/beta-catenin pathway through LRP6 in colorectal cancer. Oncogene. 2015; 34:4914-4927. [PubMed: 25500543]

14. Lu Z, Ghosh S, Wang Z, Hunter T. Downregulation of caveolin-1 function by EGF leads to the loss of E-cadherin, increased transcriptional activity of beta-catenin, and enhanced tumor cell invasion. Cancer Cell. 2003; 4:499-515. [PubMed: 14706341]

15. Hazan RB, Norton L. The epidermal growth factor receptor modulates the interaction of Ecadherin with the actin cytoskeleton. The Journal of biological chemistry. 1998; 273:9078-9084. [PubMed: 9535896] 
16. Vogelstein B, Kinzler KW. The Path to Cancer -Three Strikes and You're Out. N Engl J Med. 2015; 373:1895-1898. [PubMed: 26559569]

17. Yan J, et al. FZD6, targeted by miR-21, represses gastric cancer cell proliferation and migration via activating non-canonical wnt pathway. Am J Transl Res. 2016; 8:2354-2364. [PubMed: 27347343]

18. Ara $\mathrm{H}$, et al. Role for Daple in non-canonical Wnt signaling during gastric cancer invasion and metastasis. Cancer Sci. 2016; 107:133-139. [PubMed: 26577606]

19. Ford CE, et al. The non-canonical Wnt ligand, Wnt5a, is upregulated and associated with epithelial to mesenchymal transition in epithelial ovarian cancer. Gynecol Oncol. 2014; 134:338-345. [PubMed: 24924122]

20. Avasarala S, et al. Heterotrimeric G-protein, Galpha16, is a critical downstream effector of noncanonical Wnt signaling and a potent inhibitor of transformed cell growth in non small cell lung cancer. PLoS One. 2013; 8:e76895. [PubMed: 24204697]

21. Vela I, et al. PITX2 and non-canonical Wnt pathway interaction in metastatic prostate cancer. Clin Exp Metastasis. 2014; 31:199-211. [PubMed: 24162257]

22. Katoh M, Katoh M. Comparative integromics on non-canonical WNT or planar cell polarity signaling molecules: transcriptional mechanism of PTK7 in colorectal cancer and that of SEMA6A in undifferentiated ES cells. Int J Mol Med. 2007; 20:405-409. [PubMed: 17671748]

23. Tseng JC, et al. CAPE suppresses migration and invasion of prostate cancer cells via activation of non-canonical Wnt signaling. Oncotarget. 2016; 7:38010-38024. [PubMed: 27191743]

24. Asem MS, Buechler S, Wates RB, Miller DL, Stack MS. Wnt5a Signaling in Cancer. Cancers (Basel). 2016; 8

25. Qi H, et al. Wnt5a promotes vasculogenic mimicry and epithelial-mesenchymal transition via protein kinase Calpha in epithelial ovarian cancer. Oncol Rep. 2014; 32:771-779. [PubMed: 24898696]

26. Katoh M. Network of WNT and other regulatory signaling cascades in pluripotent stem cells and cancer stem cells. Curr Pharm Biotechnol. 2011; 12:160-170. [PubMed: 21044011]

27. Chen YS, et al. Proteomics profiling of Madin-Darby canine kidney plasma membranes reveals Wnt-5a involvement during oncogenic H-Ras/TGF-beta-mediated epithelial-mesenchymal transition. Mol Cell Proteomics. 2011; 10:M110 001131.

28. Aznar N, et al. Daple is a novel non-receptor GEF required for trimeric G protein activation in Wnt signaling. eLife. 2015; 4:e07091. [PubMed: 26126266]

29. Ishida-Takagishi M, et al. The Dishevelled-associating protein Daple controls the non-canonical Wnt/Rac pathway and cell motility. Nat Commun. 2012; 3:859. [PubMed: 22643886]

30. Barbazan J, et al. Prognostic Impact of Modulators of G proteins in Circulating Tumor Cells from Patients with Metastatic Colorectal Cancer. Sci Rep. 2016; 6:22112. [PubMed: 26916336]

31. Oshita A, et al. Identification and characterization of a novel Dvl-binding protein that suppresses Wnt signalling pathway. Genes Cells. 2003; 8:1005-1017. [PubMed: 14750955]

32. Kilander MB, et al. Disheveled regulates precoupling of heterotrimeric G proteins to Frizzled 6. FASEB J. 2014; 28:2293-2305. [PubMed: 24500924]

33. Stricker S, Rauschenberger V, Schambony A. ROR-Family Receptor Tyrosine Kinases. Curr Top Dev Biol. 2017; 123:105-142. [PubMed: 28236965]

34. Green JL, Kuntz SG, Sternberg PW. Ror receptor tyrosine kinases: orphans no more. Trends Cell Biol. 2008; 18:536-544. [PubMed: 18848778]

35. Oishi I, et al. The receptor tyrosine kinase Ror2 is involved in non-canonical Wnt5a/JNK signalling pathway. Genes Cells. 2003; 8:645-654. [PubMed: 12839624]

36. Lane JR, et al. Antibodies that identify only the active conformation of G(i) family G protein alpha subunits. FASEB J. 2008; 22:1924-1932. [PubMed: 18199696]

37. van der Gun BT, et al. EpCAM in carcinogenesis: the good, the bad or the ugly. Carcinogenesis. 2010; 31:1913-1921. [PubMed: 20837599]

38. Pace $\mathrm{CN}$, et al. Tyrosine hydrogen bonds make a large contribution to protein stability. J Mol Biol. 2001; 312:393-404. [PubMed: 11554795] 
39. Kufareva I, Ilatovskiy AV, Abagyan R. Pocketome: an encyclopedia of small-molecule binding sites in 4D. Nucleic Acids Research. 2012; 40:D535-D540. [PubMed: 22080553]

40. England P, Bregegere F, Bedouelle H. Energetic and kinetic contributions of contact residues of antibody D1.3 in the interaction with lysozyme. Biochemistry. 1997; 36:164-172. [PubMed: 8993330]

41. Kothari AN, et al. Osteopontin-A Master Regulator of Epithelial-Mesenchymal Transition. J Clin Med. 2016; 5

42. Dong Q, et al. Osteopontin promotes epithelial-mesenchymal transition of hepatocellular carcinoma through regulating vimentin. Oncotarget. 2016; 7:12997-13012. [PubMed: 26824421]

43. Yook JI, et al. A Wnt-Axin2-GSK3beta cascade regulates Snail1 activity in breast cancer cells. Nat Cell Biol. 2006; 8:1398-1406. [PubMed: 17072303]

44. Subbaiah VK, Kranjec C, Thomas M, Banks L. PDZ domains: the building blocks regulating tumorigenesis. Biochem J. 2011; 439:195-205. [PubMed: 21954943]

45. Dalerba P, et al. Single-cell dissection of transcriptional heterogeneity in human colon tumors. Nat Biotechnol. 2011; 29:1120-1127. [PubMed: 22081019]

46. Markman B, Javier Ramos F, Capdevila J, Tabernero J. EGFR and KRAS in colorectal cancer. Advances in clinical chemistry. 2010; 51:71-119. [PubMed: 20857619]

47. Radziwill G, Erdmann RA, Margelisch U, Moelling K. The Bcr kinase downregulates Ras signaling by phosphorylating AF-6 and binding to its PDZ domain. Mol Cell Biol. 2003; 23:46634672. [PubMed: 12808105]

48. Gardoni F, et al. CaMKII-dependent phosphorylation regulates SAP97/NR2A interaction. The Journal of biological chemistry. 2003; 278:44745-44752. [PubMed: 12933808]

49. Cohen NA, Brenman JE, Snyder SH, Bredt DS. Binding of the inward rectifier K+ channel Kir 2.3 to PSD-95 is regulated by protein kinase A phosphorylation. Neuron. 1996; 17:759-767. [PubMed: 8893032]

50. Liu X, Shepherd TR, Murray AM, Xu Z, Fuentes EJ. The structure of the Tiam1 PDZ domain/ phospho-syndecan 1 complex reveals a ligand conformation that modulates protein dynamics. Structure. 2013; 21:342-354. [PubMed: 23395182]

51. Seitz K, et al. beta-Arrestin interacts with the beta/gamma subunits of trimeric G-proteins and dishevelled in the $\mathrm{Wnt} / \mathrm{Ca}(2+)$ pathway in xenopus gastrulation. PLoS One. 2014; 9:e87132. [PubMed: 24489854]

52. Angers S, et al. Molecular architecture and assembly of the DDB1-CUL4A ubiquitin ligase machinery. Nature. 2006; 443:590-593. [PubMed: 16964240]

53. Egger-Adam D, Katanaev VL. The trimeric G protein Go inflicts a double impact on axin in the Wnt/frizzled signaling pathway. Dev Dyn. 2010; 239:168-183. [PubMed: 19705439]

54. Jung H, et al. Negative feedback regulation of Wnt signaling by Gbetagamma-mediated reduction of Dishevelled. Exp Mol Med. 2009; 41:695-706. [PubMed: 19561403]

55. Sugimura R, Li L. Noncanonical Wnt signaling in vertebrate development, stem cells, and diseases. Birth Defects Res C Embryo Today. 2010; 90:243-256. [PubMed: 21181886]

56. Lowery FJ, Yu D. Growth factor signaling in metastasis: current understanding and future opportunities. Cancer Metastasis Rev. 2012; 31:479-491. [PubMed: 22706845]

57. Pukrop T, Binder C. The complex pathways of Wnt 5a in cancer progression. J Mol Med (Berl). 2008; 86:259-266. [PubMed: 17952396]

58. Huang CL, et al. Wnt5a expression is associated with the tumor proliferation and the stromal vascular endothelial growth factor-an expression in non-small-cell lung cancer. J Clin Oncol. 2005; 23:8765-8773. [PubMed: 16314637]

59. Kurayoshi M, et al. Expression of Wnt-5a is correlated with aggressiveness of gastric cancer by stimulating cell migration and invasion. Cancer Res. 2006; 66:10439-10448. [PubMed: 17079465]

60. Garcia-Marcos M, Ghosh P, Ear J, Farquhar MG. A structural determinant that renders G alpha(i) sensitive to activation by GIV/girdin is required to promote cell migration. The Journal of biological chemistry. 2010; 285:12765-12777. [PubMed: 20157114] 
61. Garcia-Marcos M, Ghosh P, Farquhar MG. GIV is a nonreceptor GEF for G alpha i with a unique motif that regulates Akt signaling. Proc Natl Acad Sci U S A. 2009; 106:3178-3183. [PubMed: 19211784]

62. Ghosh P, et al. A G\{alpha\}i-GIV molecular complex binds epidermal growth factor receptor and determines whether cells migrate or proliferate. Mol Biol Cell. 2010; 21:2338-2354. [PubMed: 20462955]

63. Ghosh P, Garcia-Marcos M, Bornheimer SJ, Farquhar MG. Activation of Galphai3 triggers cell migration via regulation of GIV. J Cell Biol. 2008; 182:381-393. [PubMed: 18663145]

64. Garcia-Marcos M, Kietrsunthorn PS, Wang H, Ghosh P, Farquhar MG. G Protein binding sites on Calnuc (nucleobindin 1) and NUCB2 (nucleobindin 2) define a new class of G(alpha)i-regulatory motifs. The Journal of biological chemistry. 2011; 286:28138-28149. [PubMed: 21653697]

65. Lin C, et al. Tyrosine phosphorylation of the Galpha-interacting protein GIV promotes activation of phosphoinositide 3-kinase during cell migration. Sci Signal. 2011; 4:ra64. [PubMed: 21954290]

66. Yao R, Natsume Y, Noda T. MAGI-3 is involved in the regulation of the JNK signaling pathway as a scaffold protein for frizzled and Ltap. Oncogene. 2004; 23:6023-6030. [PubMed: 15195140]

67. Garcia-Marcos M, Ear J, Farquhar MG, Ghosh P. A GDI (AGS3) and a GEF (GIV) regulate autophagy by balancing $\mathrm{G}$ protein activity and growth factor signals. Mol Biol Cell. 2011; 22:673686. [PubMed: 21209316]

68. Longo PA, Kavran JM, Kim MS, Leahy DJ. Transient mammalian cell transfection with polyethylenimine (PEI). Methods in enzymology. 2013; 529:227-240. [PubMed: 24011049]

69. Guttman M, et al. Interactions of the NPXY microdomains of the low density lipoprotein receptorrelated protein 1. Proteomics. 2009; 9:5016-5028. [PubMed: 19771558]

70. McCormack AL, et al. Direct analysis and identification of proteins in mixtures by LC/MS/MS and database searching at the low-femtomole level. Anal Chem. 1997; 69:767-776. [PubMed: 9043199]

71. Lin C, et al. Structural basis for activation of trimeric Gi proteins by multiple growth factor receptors via GIV/Girdin. Mol Biol Cell. 2014; 25:3654-3671. [PubMed: 25187647]

72. Cardozo T, Totrov M, Abagyan R. Homology modeling by the ICM method. Proteins: Structure, Function, and Genetics. 1995; 23:403-414.

73. Zhang Y, et al. Inhibition of Wnt signaling by Dishevelled PDZ peptides. Nat Chem Biol. 2009; 5:217-219. [PubMed: 19252499]

74. Jorissen RN, et al. Metastasis-Associated Gene Expression Changes Predict Poor Outcomes in Patients with Dukes Stage B and C Colorectal Cancer. Clin Cancer Res. 2009; 15:7642-7651. [PubMed: 19996206]

75. Smith JJ, et al. Experimentally derived metastasis gene expression profile predicts recurrence and death in patients with colon cancer. Gastroenterology. 2010; 138:958-968. [PubMed: 19914252]

76. Thorsteinsson M, et al. Gene expression profiles in stages II and III colon cancers: application of a 128-gene signature. Int J Colorectal Dis. 2012; 27:1579-1586. [PubMed: 22710688]

77. Laibe S, et al. A seven-gene signature aggregates a subgroup of stage II colon cancers with stage III. OMICS. 2012; 16:560-565. [PubMed: 22917480]

78. Dalerba P, et al. CDX2 as a Prognostic Biomarker in Stage II and Stage III Colon Cancer. N Engl J Med. 2016; 374:211-222. [PubMed: 26789870]

79. Sahoo D, Dill DL, Gentles AJ, Tibshirani R, Plevritis SK. Boolean implication networks derived from large scale, whole genome microarray datasets. Genome Biol. 2008; 9:R157. [PubMed: 18973690] 
A

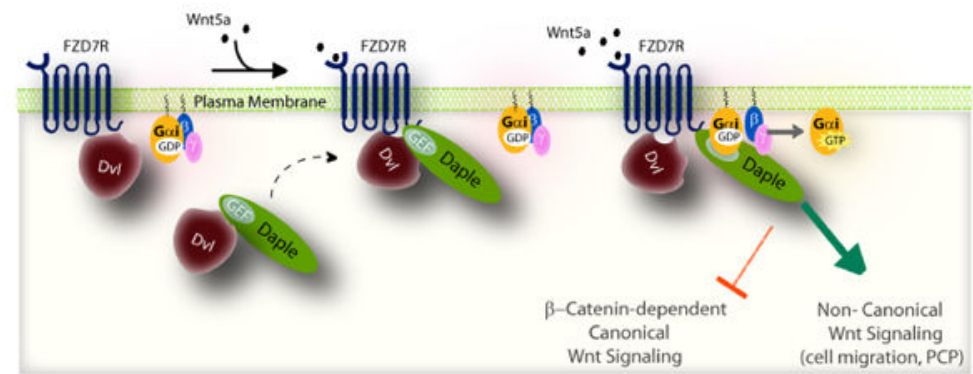

B

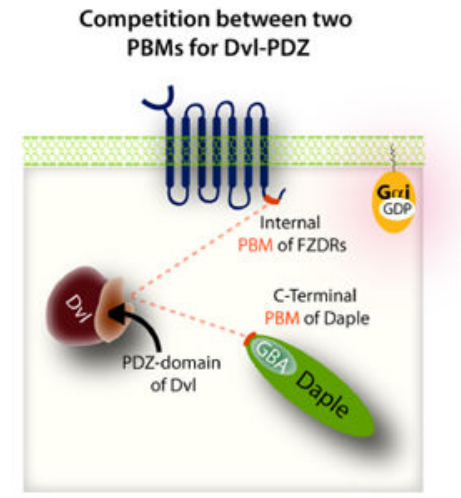

D
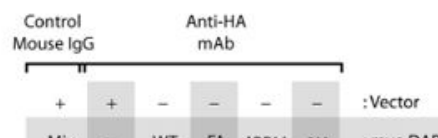

Mix - WT FA $\triangle P B M \quad 2 M \quad$ :myC-DAPLE
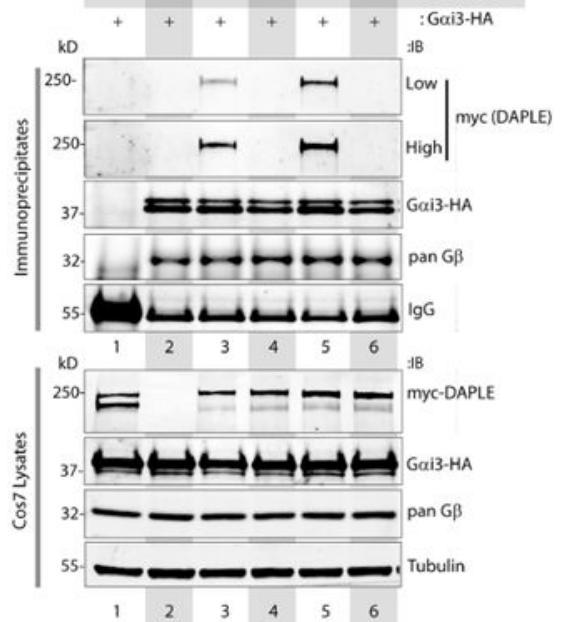

C Competition between Dvl and Gai for Daple

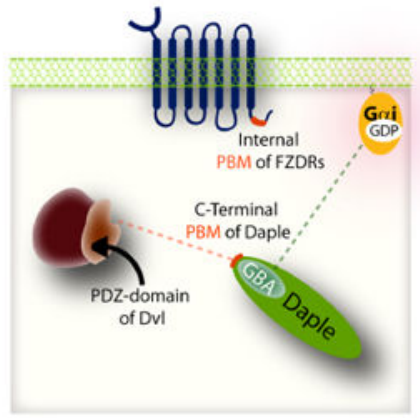

$\mathrm{E}$

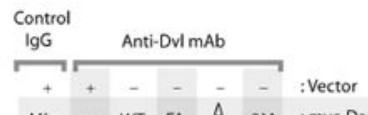

Mix - WT FA $\triangle \mathrm{DBM}_{2 \mathrm{M}}$ :myc-Daple

+++++ : Dvl1
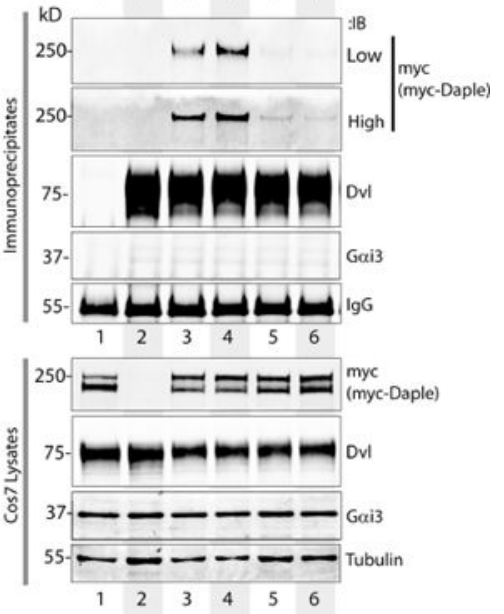

Figure 1. The $G_{a i}$-binding and activating [GBA] and PDZ-binding motifs [PBM] in Daple allosterically inhibit each other's functions

(A) Schematic summarizing how Daple enhances non-canonical Wnt signaling downstream of FZDs. (B-C) Schematic showing two key intermolecular interplays encountered during non-canonical Wnt signaling via Daple, as shown in (28) - a) FZD7's internal PBM and Daple's c-terminal PBM compete for binding to the PDZ-domain of Dvl (B). b) Dvl and Gai compete for two distinct modules on Daple; Dvl-PDZ and $\mathrm{G}_{\mathbf{a i}}$ compete for Daple (C). (D) Immunoprecipitation assay assessing the binding of $\mathrm{G}_{\mathbf{a i}}$ to full-length Daple wild-type (WT) 
or mutants [F1675A (GBA-deficient, FA), $\Delta \mathrm{PBM}$, or double mutant (FA+ $\Delta \mathrm{PBM}$; also called $2 \mathrm{M}$ )]. Bound complexes were analyzed for Daple (myc) and $\mathrm{G}_{\mathbf{a} \mathbf{3} 3}$ (HA). $\mathrm{G} \beta$ was monitored as a positive control for $\mathrm{G}_{\mathbf{a i} 3}$-bound proteins. Quantification of blots is shown in Fig. S1A.

(E) As in (D), Immunoprecipitation assay assessing binding of Dvl to various Daple mutants in 1D. Quantification of blots is shown in Fig. S1B. 
A

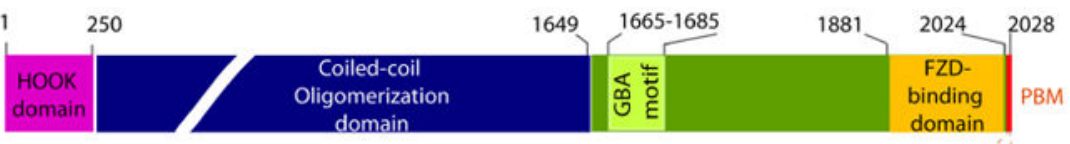

HUMAN DAPLE COW_DÄPLE MOUS̄E_DAPLE RAT_DAPPL CHICKEN DAPLE ZEBRAFISH DAPLE
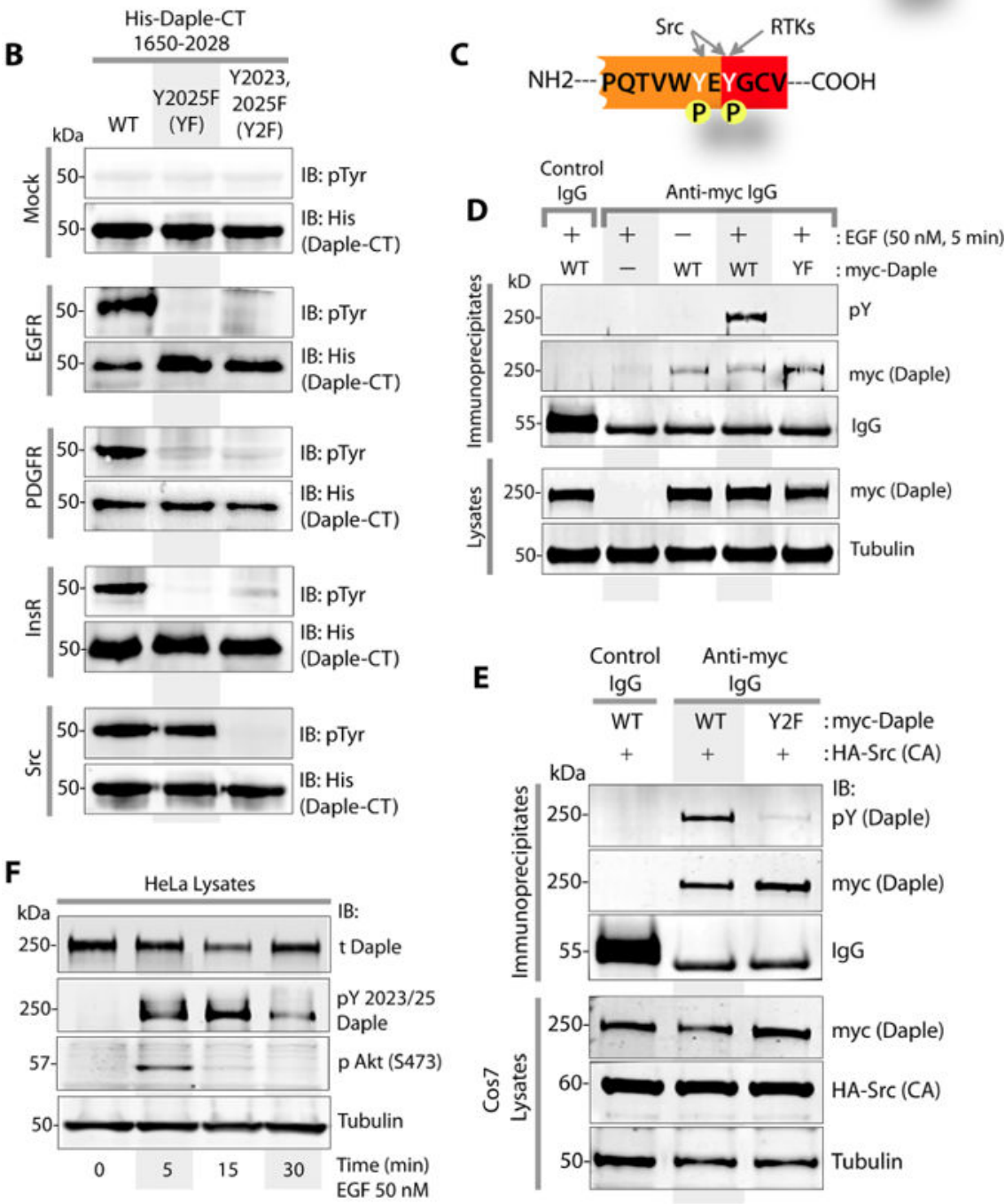

Figure 2. Multiple TKs phosphorylate Daple's C-terminal PBM module

(A) Top: Schematic showing the domain arrangement of Daple. Bottom: Alignment of Daple's C-terminus in various species displaying an evolutionary conserved PDZ-binding motif [PBM; red box]. The two phosphotyrosines defined in this work are highlighted in yellow. (B-C) Daple is phosphorylated in vitro by both RTKs and the non-RTK, Src. In vitro kinase assays were carried out using several recombinant kinases and wild-type (WT) or non-phosphorylatable mutants [Y2025F (YF) and Y2023/2025F (Y2F)] His-Daple CT proteins as substrate. Reactions were analyzed for phosphotyrosine-Daple with anti-pTyr Ab 
(B). Schematic in C summarizes findings in B. (D-F) Daple is phosphorylated on tyrosines in cells. Immunoprecipitation assays (D) assessing the phosphorylation of Daple after EGF stimulation in Cos7 cells co-expressing EGFR and myc-Daple [WT or YF mutant]. Immune complexes were analyzed for phosphorylated (pan-pTyr) and total (myc) Daple. (E)

Immunoprecipitation assay assessing the phosphorylation of Daple by Src in Cos7 cells coexpressing a constitutively active (CA; Y527F) Src mutant and myc-Daple [WT or Y2F mutant]. Immunoblotting were performed as in D. (F) Tyrosine phosphorylation of endogenous Daple was assessed in HeLa cells responding to EGF. Whole cell lysates were analyzed for pTyr-Daple with anti-pYDaple (pY2023, pY2025; see Fig. S2 for antibody validation) and phosphorylation of Akt (pS473). 
A

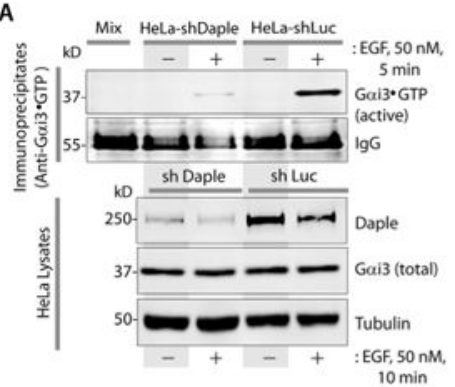

D

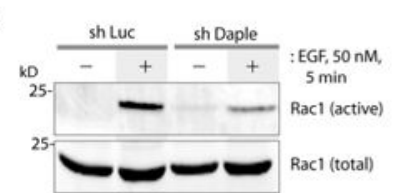

E

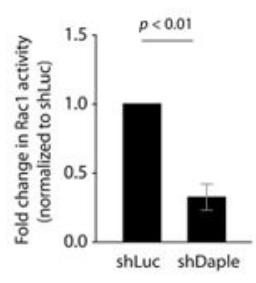

H
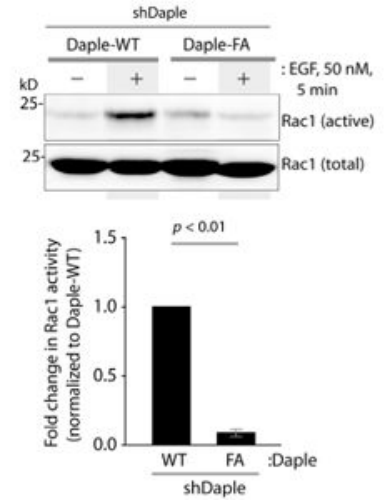

J

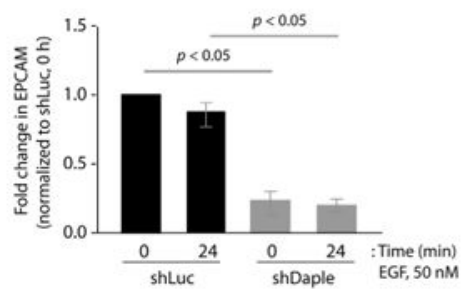

B

C

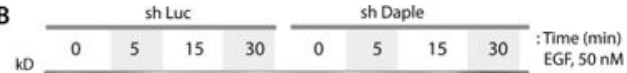

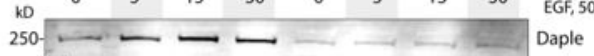

95- - - - - - - - -catenin
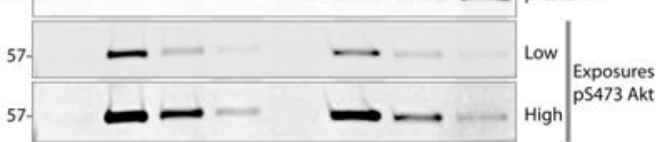

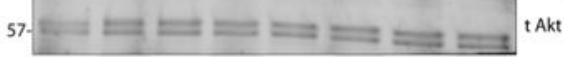

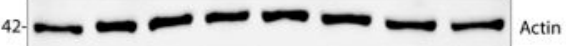
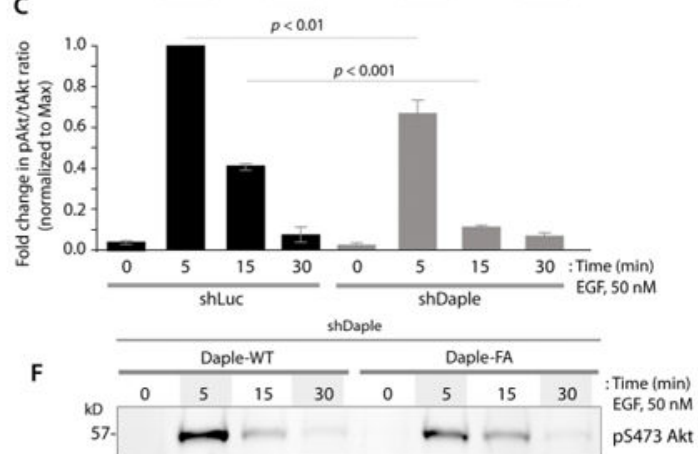

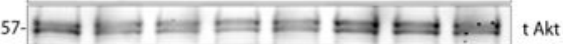

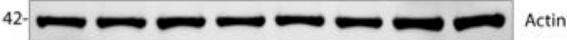

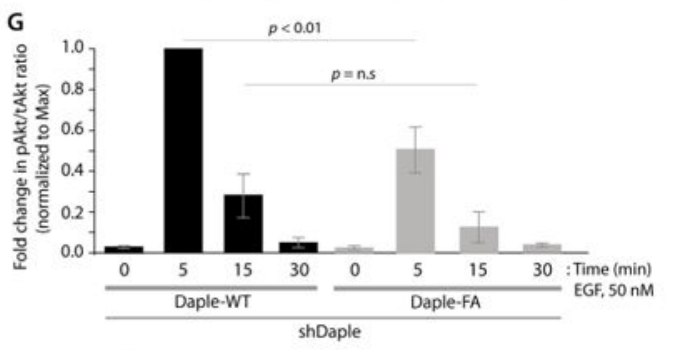

K

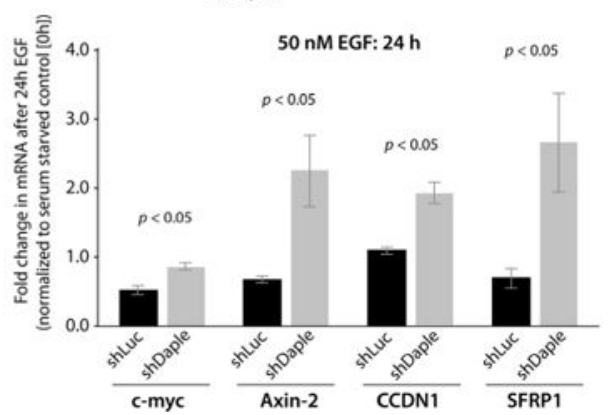

Figure 3. Daple is required for activation of $G_{a i 3}$, Rac1 and Akt signals and in the antagonistic inhibition of $\beta$-catenin-dependent Wnt signals downstream of EGF/EGFR

(A) Immunoprecipitation assay assessing $G_{\mathbf{a i} 3}$ activation after EGF stimulation in control (shLuc) and Daple-depleted (shDaple) HeLa cells. Western blot for active $\mathrm{G}_{\mathbf{a i 3}}\left(\mathrm{G}_{\mathbf{a i 3}}-\mathrm{GTP}\right)$ and total $\mathrm{G}_{\mathbf{a i 3}}$. Quantification of blots is shown in Fig. S3. (B) Whole cell lysates of EGFstimulated HeLa cells in A were analyzed for phosphorylation of Akt (S473), Daple, $\beta$ catenin, and Actin (loading control). (C) Bar graphs display quantification of phosphorylated/total Akt. Error bars represent mean \pm S.D; $n=3$. (D) Lysates of HeLa cells 
in A were analyzed for Rac1 activity (see Methods) after EGF stimulation. (D) Quantification of Rac1 activation is displayed as bar graphs. Error bars represent mean \pm S.D; n=3. (E) (F) Whole cell lysates of EGF-stimulated HeLa cells expressing Daple-WT or Daple-F1675A (FA) mutant were analyzed for Akt phosphorylation as in B. (G) Bar graphs display quantification of phosphorylated/total Akt. Error bars represent mean \pm S.D; n=3. (H) Lysates of HeLa cells in F were analyzed for Rac1 activity (see Methods) after EGF stimulation. Phosphorylation of Daple coincides with the onset of Akt phosphorylation and peaks at 15 min after EGF stimulation. (I) Quantification of Rac1 activation is displayed as bar graphs. Error bars represent mean $\pm \mathrm{S}$.D; $\mathrm{n}=3$. (J-K) qPCR analysis assessing EPCAM (J) and other Wnt target genes (K) induced by EGF in HeLa cells used in A. Bar graphs display the fold change in RNA (y axis) after EGF stimulation. Error bars represent mean \pm S.D; $\mathrm{n}=3$. 
A
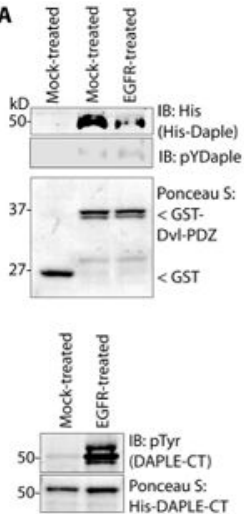

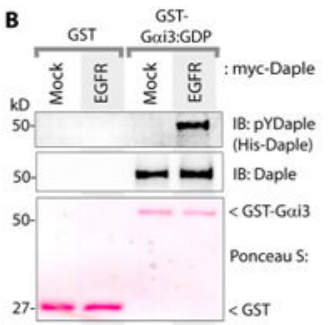

c

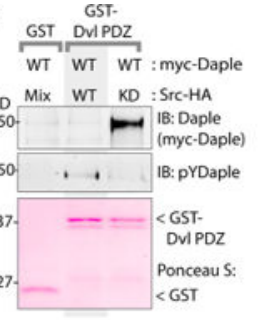

D

GST GST. WT WT WT : myc-Daple KD Mix WT KD : SrC-HA 250. - - 18: Daple 250- $\quad$ 18: pYDaple
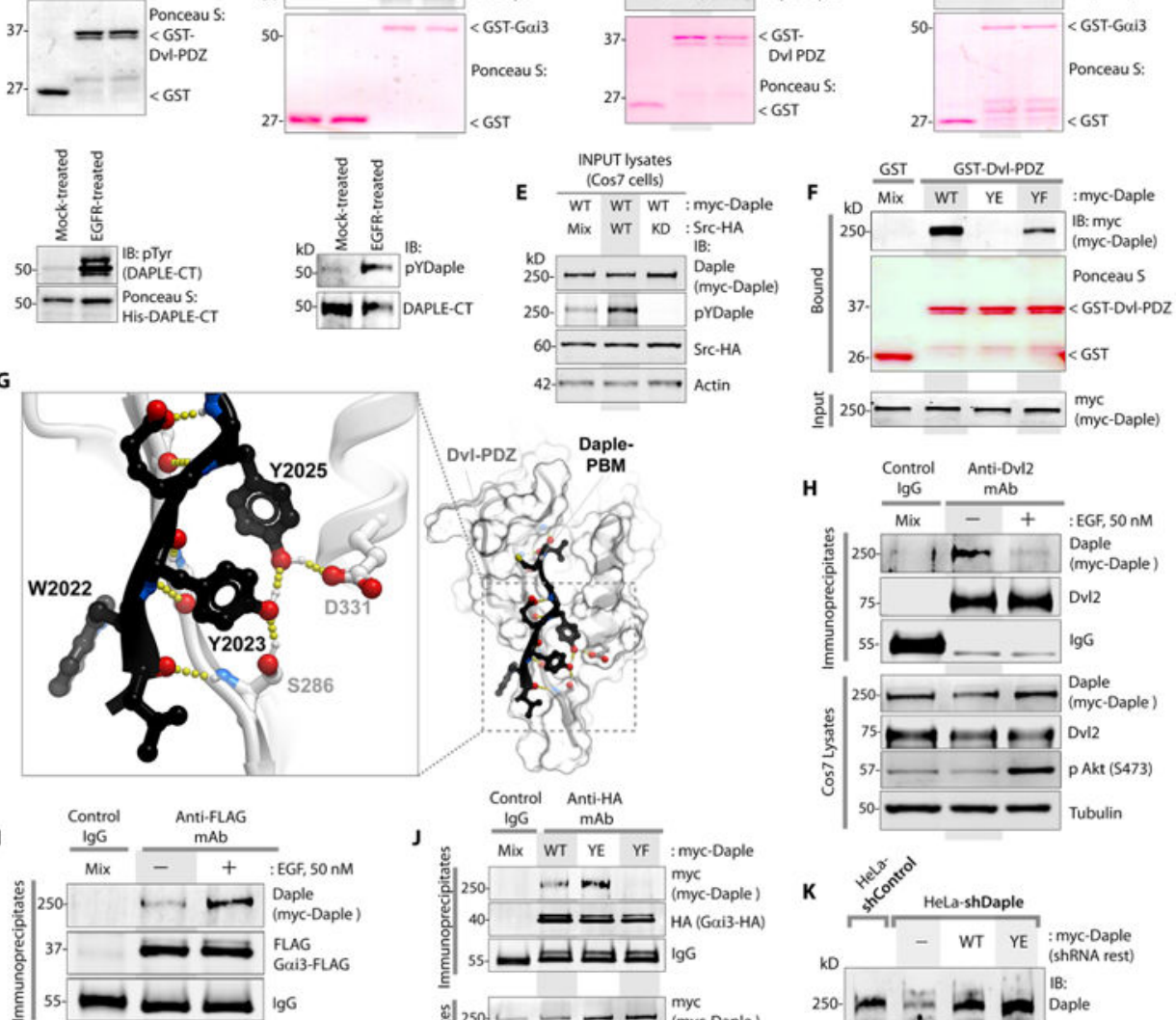

J

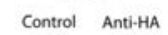

IgG Anti-HA
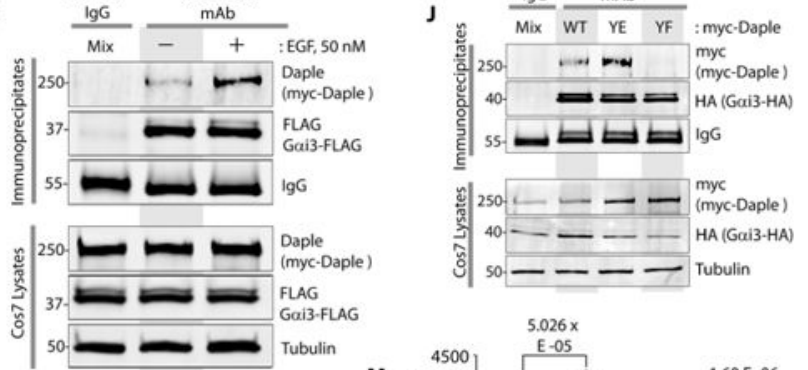

L
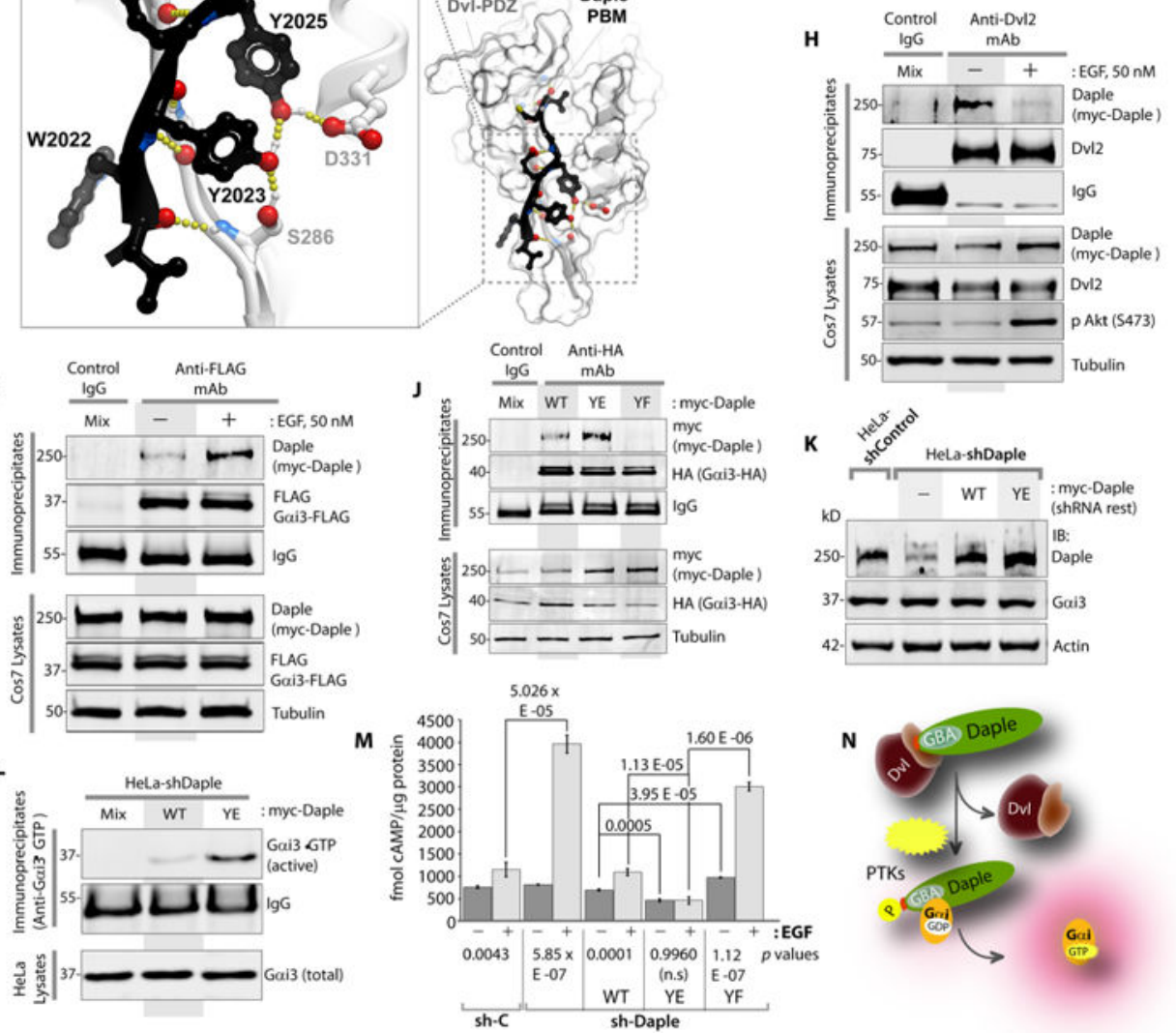

N

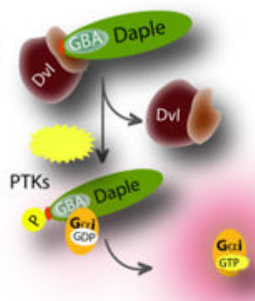

Figure 4. Tyrosine phosphorylation inhibits Daple's ability to bind Dvl, but favors binding to and activation of $\mathbf{G}_{\text {ai3 }}$

(A-B) GST pulldown assays investigating the impact of phosphorylation of Daple by EGFR on binding to Dvl (A) and $\mathrm{G}_{\mathbf{a i 3}}$ (B). Bound total and phospho-Daple (pYDaple, as determined using an anti-pY2023/25 Daple Ab) were analyzed by immunoblotting and quantified (Fig. S4A-B). (C-E) GST pulldown assays assessing the impact of in cellulo phosphorylation of Daple by Src on binding to Dvl (C) and $\mathrm{G}_{\mathbf{a i 3}}$ (D). Bound total (myc) and phospho-Daple (pYDaple) were analyzed by immunoblotting as in A-B and quantified (Fig. 
S4C). (F) GST pulldown assays investigating the impact of phosphomimicking (YE) and non-phosphorylatable (YF) mutants of Daple on binding to Dvl. Bound Daple (myc) was analyzed by immunoblotting and quantified (Fig. 4E). (G) Homology-based model of Daple's PBM (black ribbon and sticks) bound to Dvl2-PDZ domain (grey surface) was generated using solved crystal structures of Dvl2-PDZ bound to multiple peptides [see Methods]. Each tyrosine is predicted to form H-bonds (yellow dotted lines) with each other and with Dvl2 (D331 and S286). (H-I) Immunoprecipitation assays assessing the impact of EGF stimulation on the abundance of Daple:Dvl (H) or Daple: $\mathrm{G}_{\mathrm{ai} 3}$ (I) complexes in Cos7 cells. Dvl- or $\mathrm{G}_{\mathrm{ai} 3}$-bound immune complexes were analyzed for Daple (myc), and fold changes in binding were quantified (Fig S4F-G). (J) Immunoprecipitation assays assays investigating the impact of phosphomimicking (YE) and non-phosphorylatable (YF) mutants of Daple on binding to $\mathrm{G}_{\mathrm{ai} 3}$. $\mathrm{G}_{\mathrm{ai} 3}$-bound immune complexes were analyzed for Daple as in $\mathrm{H}$ and quantified (Fig. S4H). (K) Whole-cell lysates from control (shControl) or Dapledepleted (shDaple) HeLa cells stably expressing Daple-WT and YE were analyzed for Daple, $\mathrm{G}_{\mathrm{ai} 3}$ and actin by immunoblotting. (L) HeLa cells in $\mathrm{K}$ were analyzed for activation of $\mathrm{G}_{\mathrm{ai} 3}$ as in $3 \mathrm{~A}$ and quantified (Fig. S4K). (M) HeLa cells expressing Daple-WT or mutants were pre-treated with forskolin and IBMX (see Methods) and treated (+) or not (-) with $50 \mathrm{nM}$ EGF (30 min) before analyzing cAMP levels by radioimmunoassay. Bar graphs display cAMP concentrations. Mean \pm S.D; $\mathrm{n}=3$. 
A

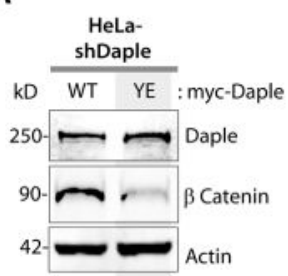

C

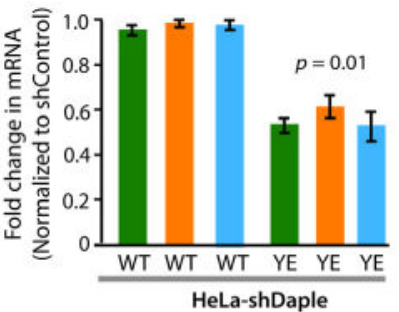

G

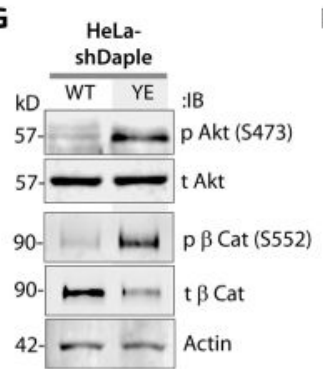

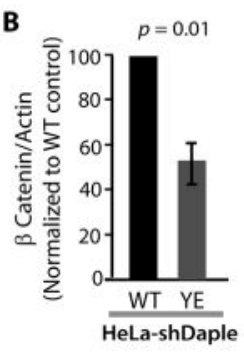
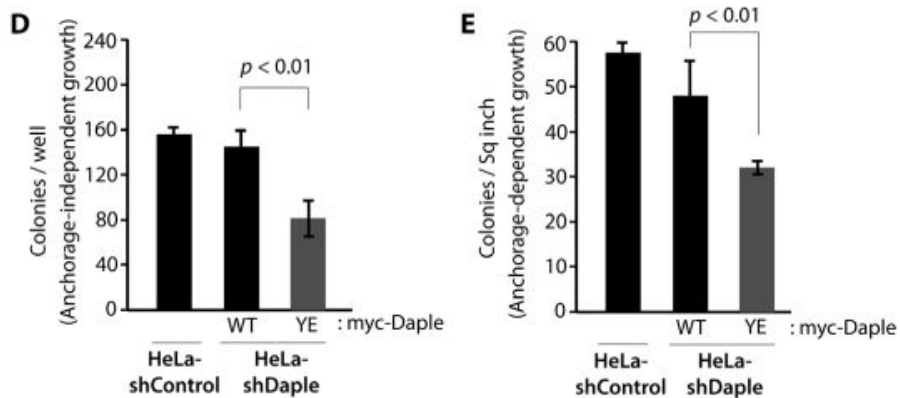

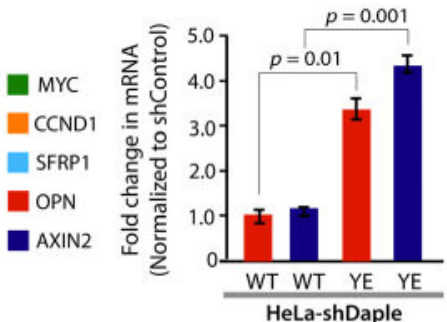

$\mathbf{F}$

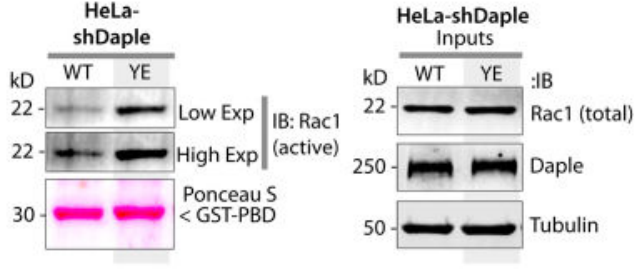

I

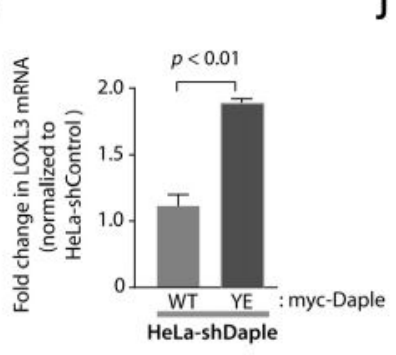

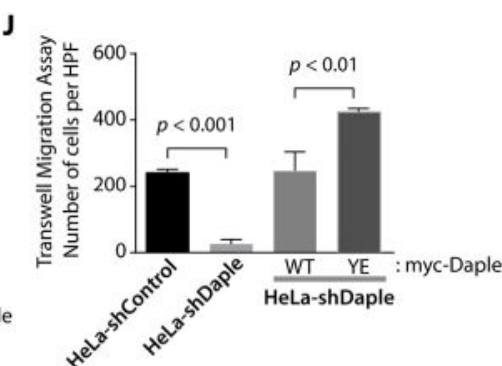

Figure 5. Tyrosine phosphorylated Daple enhances non-canonical Wnt signals, triggers EMT and cell migration

(A-B) Daple-depleted HeLa cells stably expressing Daple-WT or Daple-Y2025E (YE) were analyzed for expression of $\beta$-catenin by immunoblotting (A) and quantified and displayed as bar graphs (B). Error bars represent mean \pm S.D; $n=3$. (C) Canonical Wnt responsive $\beta$ catenin/TCF/LEF target genes were alalyzed by qPCR in HeLa cells used in A. Bar graphs display the fold change in each RNA (y axis). Error bars represent mean \pm S.D; $n=3$. (D-E) Anchorage-independent (D) and anchorage-dependent (E) colony growth assays from HeLa cells used in A (see also Fig. S5D-G). Bar graphs display the number of colonies (y axis) seen in each cell line. Error bars represent mean \pm S.D; $n=3$. (F) Rac1 activity was analyzed in HeLa cell lines used in A. (G) Western blot analysis for phospho (pS473) Akt and phospho (pS552) $\beta$-catenin from HeLa cell lines used in A. (H-I) EMT markers, Vimentin and LOXL3, were analyzed by qPCR in HeLa cell lines used in A. Bar graphs display the fold change in each mRNA ( $\mathrm{y}$ axis) normalized to the expression in cells expressing vector control. Error bars represent mean \pm S.D; $n=3$. (J) Chemotaxis was analyzed in transwell assays on HeLa cell lines used in A. Representative images are shown in Fig. S5H. Bar graphs display the number of cells/high power field (HPF); data are presented as mean \pm S.D; $n=3$. 
A

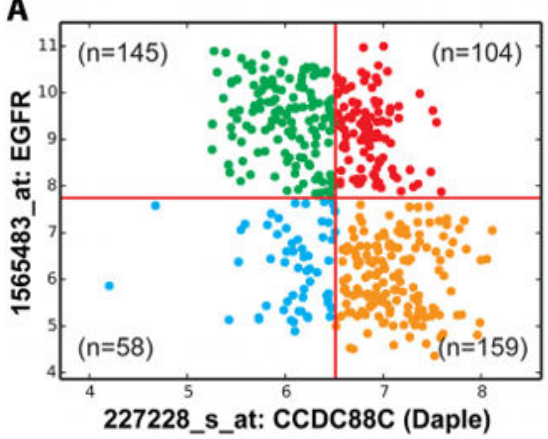

B

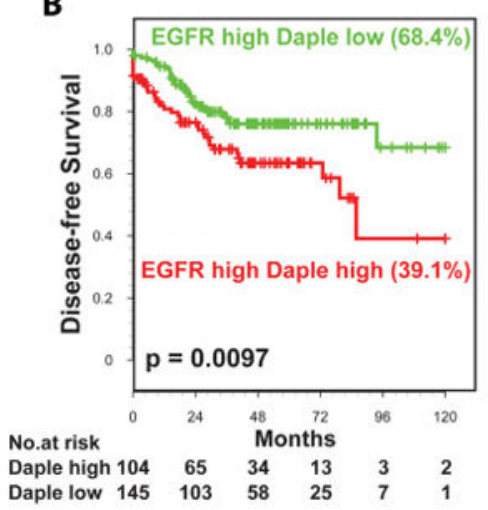

C

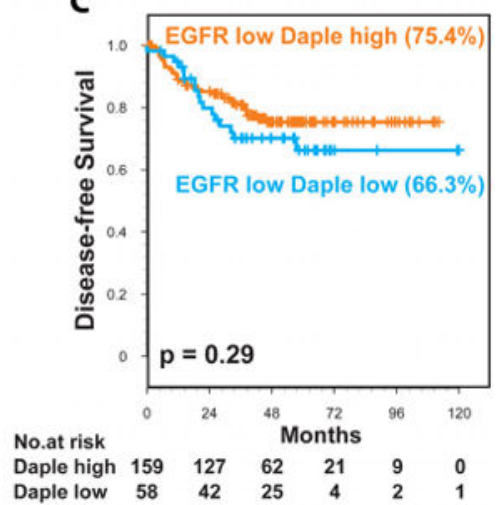

D

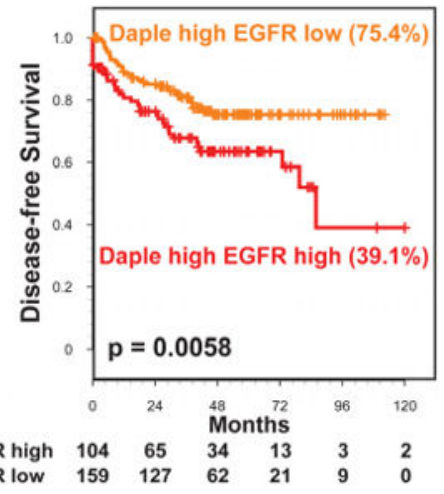

E

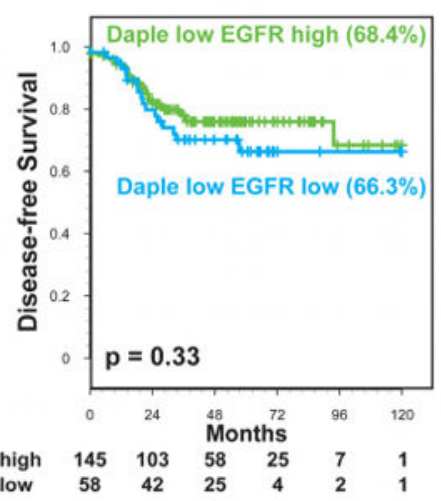

$\mathbf{F}$

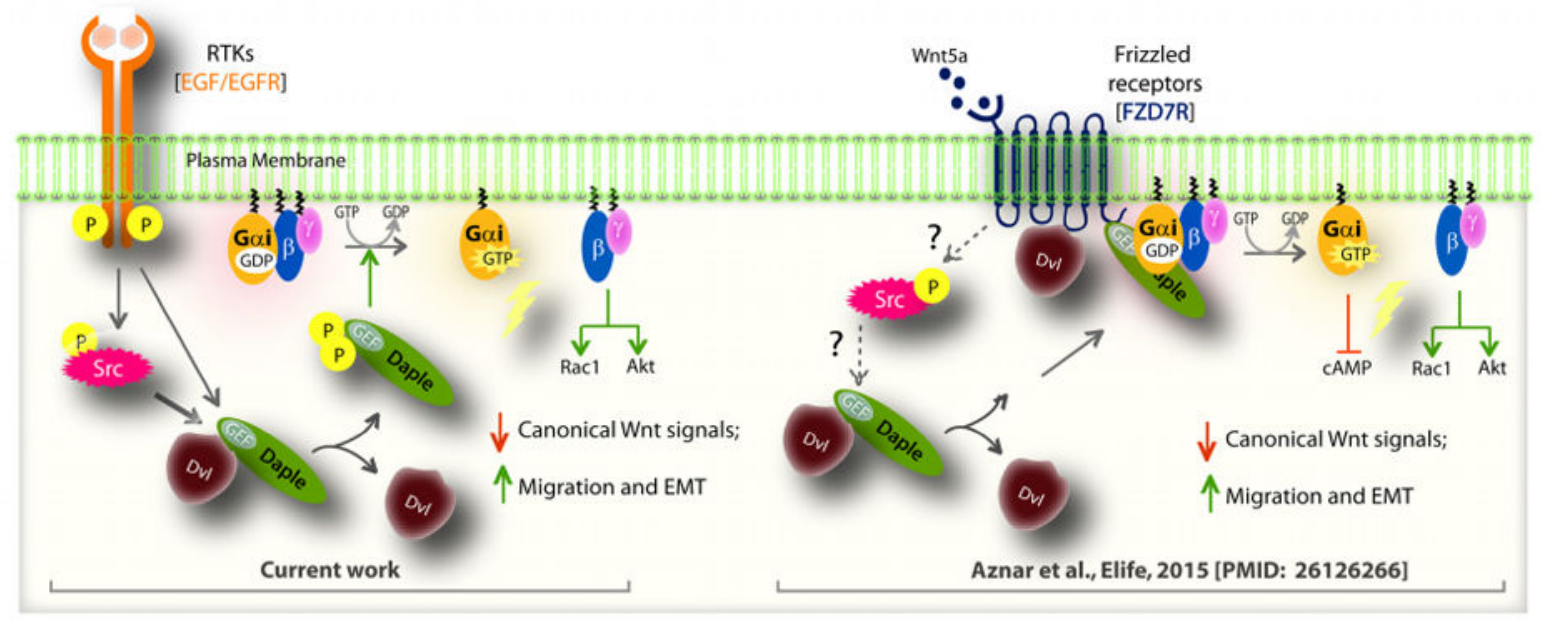

Figure 6. Concurrent expression of Daple (CCDC88C) and EGFR mRNA at high levels in colon cancer is associated with reduced disease-free survival (DFS)

(A) Graph displaying individual arrays according to the expression levels of EGFR and Daple $(C C D C 88 C)$ in a data set containing 466 patients with colon cancer. $(\mathbf{B}, \mathbf{C})$ Survival analysis using Kaplan-Meier curves displaying the impact of varying levels of Daple expression within high vs. low EGFR groups (D, E) Survival analysis using Kaplan-Meier curves displaying the impact of varying levels of Daple expression within high vs. low Daple groups. (F) Schematic of working model based on findings in current work [left] and prior 
work [right; (28)]. Left. In the absence of growth factors or Src activation, Dvl remains complexed to Daple, and $\mathrm{G}_{\mathrm{ai}}$ is largely inactive. Stimulation with growth factors like EGF triggers phosphorylation of Daple's PBM [either directly by EGFR or indirectly via activation of the non-RTK Src]. Such phosphorylation triggers dissociation of Dvl:Daple complexes and favors the assembly of Daple: $\mathrm{G}_{\mathrm{ai}}$ complexes and subsequent activation of $\mathrm{G}_{\mathrm{ai}}$. Right: Schematic summarizing the key events triggered by Wnt5a ligand demonstrated previously (28). 